\title{
Benzosultam synthesis exploiting sequential palladium-catalysed intermolecular aminosulfonylation and intramolecular sulfamidation
}

\author{
Charlotte S. Richards-Taylor, and Michael C. Willis* \\ Department of Chemistry, University of Oxford, Chemistry Research Laboratory, Mansfield Road, Oxford, OX1 3TA, UK.
}

This paper is dedicated to the memory of Professor Jonathan Williams, who was a truly inspiring colleague and friend.

\section{ARTICLE INFO}

Article history:

Received

Received in revised form

Accepted

Available online

Keywords:

Sulfonamide

Palladium catalysis

Sultam

Amination
ABSTRACT

We report a route to benzosultams that exploits the palladium catalysed aminsulfonylation of alkenyl iodides as the initial step. An $(Z)$-configured alkenyl iodide substrate is combined with DABSO, a $N, N$-dialkylhydrazine nucleophile and a palladium $(0)$ catalyst to achieve aminsulfonylation. A second palladium(0)-catalysed transformation, this time intramolecular, leads to benzosultam formation. Good variation of the starting alkenyl iodides is possible. A related $\mathrm{S}_{\mathrm{N}} \mathrm{Ar}$ route was also explored, but was shown to be less efficient.

\section{Introduction}

The sulfonamide functional group is often used as an amide replacement in biological settings due to its unique physicochemical properties. ${ }^{1}$ Cyclic sulfonamides, named sultams, are an important subclass of these compounds, and benzosultams with an aryl-N-SO $\mathrm{SO}_{2}$ linkage (benzothiazine 2,2dioxides) are far less explored in their biological relevance compared to the regioisomer with an aryl- $\mathrm{SO}_{2}-\mathrm{N}$ linkage (Figure 1a). However, there is growing interest in this structure with reports of its use as a reverse transcriptase (RT) inhibitor, ${ }^{2}$ an interleukin-8 receptor antagonist, ${ }^{3}$ and a factor Xa inhibitor (Figure1b). ${ }^{4}$

There are only a handful of methods towards the synthesis of benzothiazine 2,2-dioxides, most of which primarily feature multistep sequences and lack functional group tolerance, consequently resulting in limited substrate scope. The first known synthesis of a benzothiazine 2,2-dioxide was reported in 1966 by two groups, Loev et al. and Rossi et al., both describing a similar 7-step procedure from a sulfoacetic acid. ${ }^{5}$ More recently, a report by Mindville and co-workers described the synthesis of benzothiazine 2,2-dioxides by implementing a ring-closing metathesis, ${ }^{6}$ with the synthesis of the cyclisation substrate again requiring several synthetic steps. Literature examples for the synthesis of related derivatives, such as dibenzosultam, ${ }^{7}$ dihydrosulfostyril ${ }^{8}$ and heteroarylsultams, ${ }^{9}$ also predominately involve either multi-step procedures from a sulfonyl chloride or are restricted in the functionality of the scaffold due to the reagents employed.

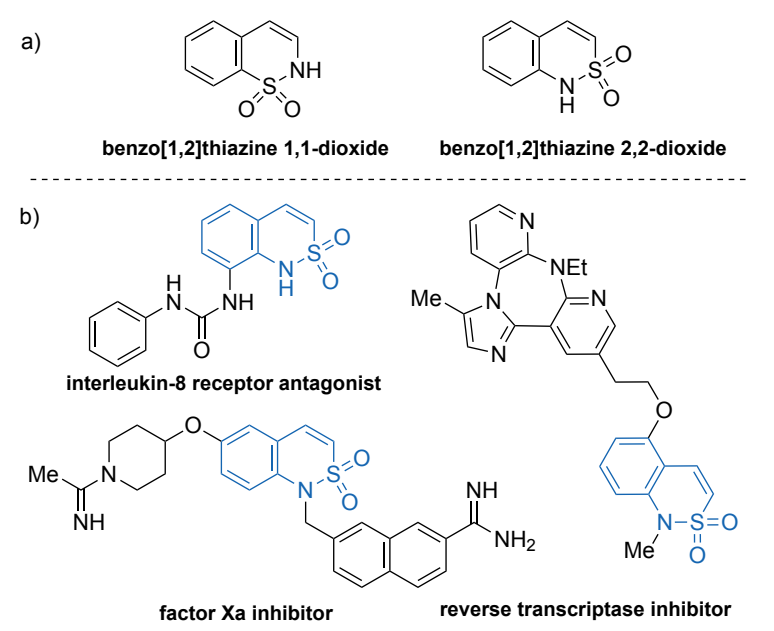

Figure 1. a) Benzosultams with aryl-N-SO $\mathrm{SO}_{2}$ and aryl- $\mathrm{SO}_{2}-\mathrm{N}$ linkages; b) Biologically relevant examples of sultams with an aryl-N-SO arrangement.

Aiming to develop a more general route to the desired benzosultams, we drew inspiration from the report from our laboratory describing a tandem palladium catalysed aminocarbonylation/amidation sequence to obtain 2-quinolones (Scheme 1a). ${ }^{10}$ We speculated that a similar approach, but employing sulfur dioxide in place of carbon monoxide, would lead to the desired benzosultams (Scheme 1b). Herein, we report the successful realisation of a variant of this approach. 
a) Aminocarbonylation/amidation route to quinolones

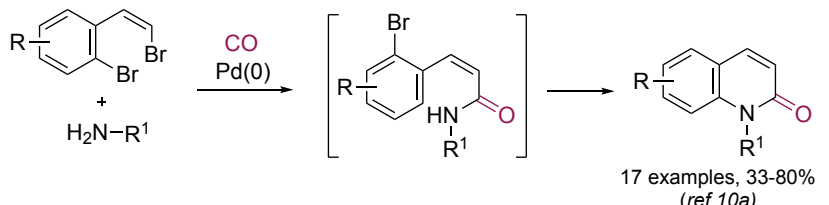

b) This work: aminosulfonylation/sulfamidation route to sultams

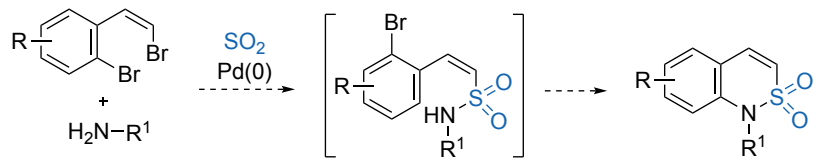

Scheme 1. a) Pd-catalysed aminocarbonylation route to 2quinolones; b) Proposed route to benzosultams.

\section{Results and discussion}

Our laboratory, ${ }^{11}$ as well as others, ${ }^{12}$ have developed a variety of palladium-catalysed sulfonylation processes, but examples that employ aryl or alkenyl halides as substrates, and proceed directly to sulfonamide products are limited to variants that use $N, N$ dialkylhydrazines as coupling partners. ${ }^{11 a, b, 12 b}$ Using our earlier 2quinolone methodology as a guide, ${ }^{10}$ and with the knowledge that palladium-catalysed aminosulfonylation reactions perform better with aryl iodide substrates, ${ }^{11 \mathrm{~b}}$ we selected alkenyl iodide $\mathbf{1 a}$ as our test substrate. Accordingly, we combined alkenyl iodide 1a with $\mathrm{N}$-aminomorpholine, DABSO (as an $\mathrm{SO}_{2}$ surrogate), ${ }^{13} \mathrm{Pd}(\mathrm{OAc})_{2}$ and CataCXium $\mathrm{A}$ as ligand, in dioxane at $70{ }^{\circ} \mathrm{C}$, which are the optimised reaction conditions from our earlier studies. ${ }^{11 \mathrm{~b}, \mathrm{c}}$ Unfortunately, none of the desired benzosultam product (4a) was isolated, but alkenyl sulfonamide 3a was obtained in 74\% yield (Scheme 2). The starting alkenyl iodide was used as a $>20: 1$ mixture of $Z: E$ isomers, and it was notable that alkenyl sulfonamide 3a was obtained with $Z: E$ of only 2.4:1. Disappointing as the failure to achieve cyclisation was, this erosion of $Z$ : $E$ ratio was also concerning, as our earlier 2-quinoline work had established that only the $Z$-isomers underwent efficient palladium-catalysed cyclisation. ${ }^{10}$ A brief survey of reaction conditions established that prolonged reaction times were problematic. A solution to this issue was found with the use of TBAI (tetrabutylammonium iodide) as an additive; the use of one equivalent of TBAI allowed the reaction time to be reduced to 3 hours, and for $N$-aminosulfonamide 3a to be isolated in $75 \%$ yield and with a $Z: E$ ratio of $12: 1$.

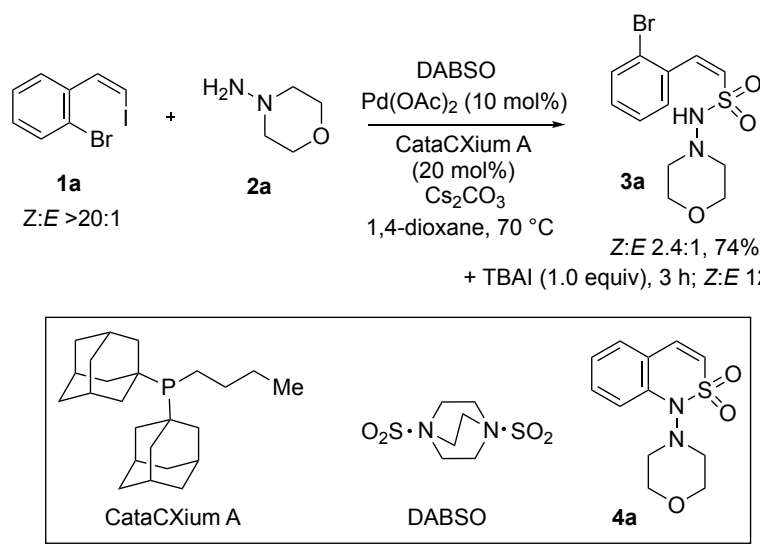

Scheme 2. Initial reactions using alkenyl iodide 1a, leading to Naminosulfonamide $\mathbf{3 a}$.
With the efficient preparation of alkenyl sulfonamide 3a in place, we tuned our attention to developing conditions for cyclisation. With the goal of achieving a single-step aminosulfonylation/cyclisation in mind, we focused on developing cyclisation conditions that were similar to the optimised aminosulfonylation process. Pleasingly, when alkenyl sulfonamide 3a was reacted with $\mathrm{Pd}(\mathrm{OAc})_{2}$ and CataCXium $\mathrm{A}$, smooth conversion to benzosultam 4a was achieved in $83 \%$ yield (Scheme 3 ). Importantly, a temperature of $100{ }^{\circ} \mathrm{C}$ was needed to achieve an efficient reaction, as performing the reaction at $70{ }^{\circ} \mathrm{C}$ delivered only a 5\% yield of benzosultam $4 a$. Returning to the desired singlestep approach, we first attempted to pause the process only for a work-up and filtration of the intermediate alkenyl sulfonamide 3a, with the crude material being subjected directly to the cyclisation conditions. Unfortunately, this approach was unsuccessful, with a maximum of $13 \%$ yield of benzosultam $4 \mathbf{a}$ being achieved.<smiles>O=S(=O)(/C=C\c1ccccc1Br)NN1CCOCC1</smiles>

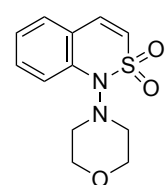

4a, $83 \%\left(5 \%\right.$ at $\left.70{ }^{\circ} \mathrm{C}\right)$

Scheme 3. Palladium-catalysed cyclisation of $N$ aminosulfonamide 3a, to benzosultam $\mathbf{4 a}$.

As an alternative to a palladium-catalysed cyclisation, we considered that with an appropriate substrate an $\mathrm{S}_{\mathrm{N}} \mathrm{Ar}$ cyclisation might be feasible. Accordingly, ortho-fluoro alkenylsulfonamide 6a was prepared using the developed conditions, starting from the corresponding F-substituted starting material (5a) (Scheme 4). However, cyclisation of sulfonamide $\mathbf{6 a}$ was not efficient, and only a poor yield (27\%) of the desired sultam (4a) could be achieved. A more efficient cyclisation was possible if a strongly electronwithdrawing trifluoromethyl substituent was added to the substrate, allowing benzosultam $\mathbf{4 b}$ to be isolated in $88 \%$ yield from the cyclisation of alkenylsulfonamide $\mathbf{6 b}$. As with our earlier approach, it was not possible to achieve sulfonylation and cyclisation in a single step using either substrate $\mathbf{5 a}$ or $\mathbf{5 b}$.
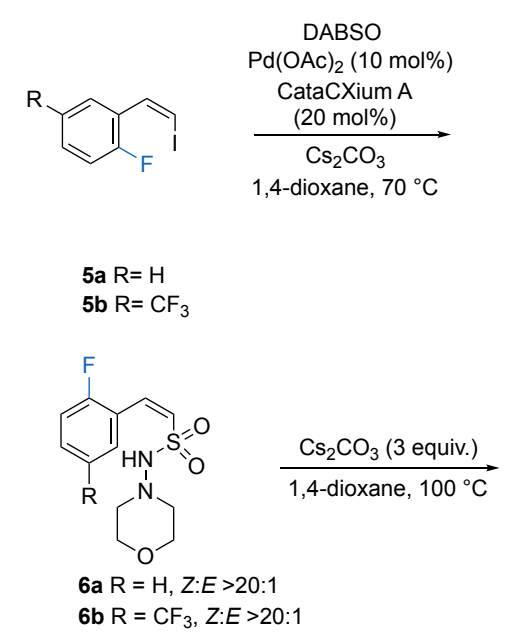

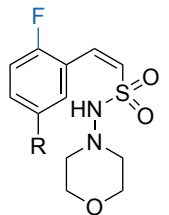

6a $Z: E 4: 1,56 \%$ 6b $Z: E 20: 1,45 \%$

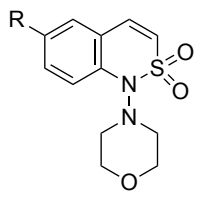

a $27 \%$ 4b $88 \%$
Scheme 4. $\mathrm{S}_{\mathrm{N}} \mathrm{Ar}$ cyclisation routes to benzosultams $\mathbf{4 a}$ and $\mathbf{4 b}$.

Given the requirement for an activating electron-withdrawing group to achieve efficient $S_{N} A r$ reactions, we reverted to a palladium-catalysed transformation to achieve cyclisation. With a one-step sulfonylation/cyclisation not possible, we then set about exploring the scope of the developed two-step route to 
benzothiazine 2,2-dioxides (Table 1). The required alkenyl iodides (1) were prepared using Wittig chemistry, as previously described, ${ }^{10 a, 14}$ and were subjected to the optimized reaction conditions.

Table 1. Two-step route to benzosultams $\mathbf{4}$, starting from alkenyl iodides $\mathbf{1}$ and proceeding via alkenylsulfonamides 3 .

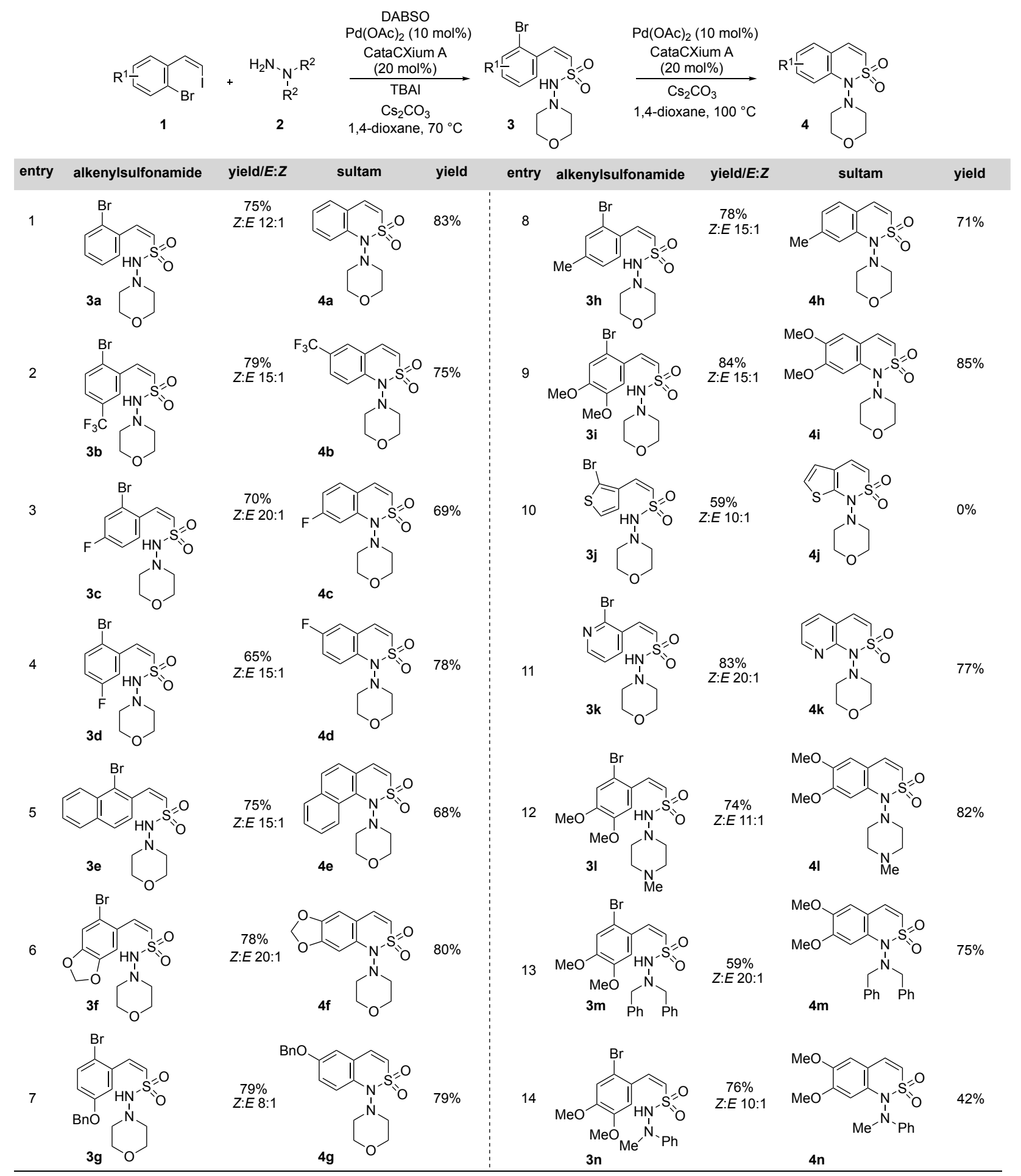

${ }^{\text {a }}$ Reaction conditions: (i) 2-(2-iodoalkenyl)aryl halide (1 equiv.), Pd(OAc) 2 (10 mol\%), CataCXium A (20 mol\%), DABSO (1.1 equiv.), TBAI (1 equiv.), 4 aminomorpholine (1.5 equiv.), 1,4-dioxane $[0.15 \mathrm{M}], 70^{\circ} \mathrm{C}, 3-4 \mathrm{~h}$; (ii) Alkenyl $\mathrm{N}$-aminosulfonamide (1 equiv.), $\mathrm{Pd}(\mathrm{OAc}) 2$ (10 mol\%), CataCXium A (20 mol\%), $\mathrm{Cs}_{2} \mathrm{CO}_{3}, 100{ }^{\circ} \mathrm{C}, 1 \mathrm{~h}$. Isolated yields.

As expected, a small drop in $Z: E$ ratio was observed for most examples during the initial palladium-catalysed aminosulfonylation step, but the alkenyl $\mathrm{N}$-aminosulfonamides were generally obtained in moderate to high yields, and with good $Z: E$ selectivity. The lowest $Z: E$ ratio was obtained with the benzyl ether variant, but was still measured at 8:1 (entry 7). After purification of the alkenyl $N$-aminosulfonamides by column chromatography, they were subjected to the cyclisation reaction conditions. The key palladium-catalysed cyclisation step worked well, and was able to deliver sultams featuring a variety of aryl and 
$\mathrm{N}$-substituents. For example, trifluoromethyl and fluoro aryl substituents were well tolerated (entries 2-4). Substrates bearing electron-donating substituents on the aryl ring also performed well (entries 6-9). Pleasingly, two heteroaryl alkenyl iodides gave the corresponding aminosulfonylation products in good yields, however, only the pyridyl example was successfully cyclised, with the thiophenyl variant failing (entries 10 and 11). Three additional hydrazine nucleophiles were explored; all delivering the expected products (entries 12-14). However, a significant drop in yield was observed for the N-methyl-N-phenyl hydrazine example, presumably due to the increased steric hindrance of the hydrazine (entry 14).

With a range of $\mathrm{N}$-amino benzosultams available we briefly explored derivatisation of these structures. Reduction of the C-C double bond in benzosultam 4a was achieved with a balloon pressure of hydrogen over a $\mathrm{Pd} / \mathrm{C}$ catalyst, to deliver the dihydroderivative 7 in excellent yield (Scheme 5). Cleavage of the N-N bond, required to deliver the parent sultam scaffold, was achieved using our previously reported procedure; ${ }^{11 \mathrm{~b}}$ hydrogenolysis of the benzyl groups in $\mathrm{N}$-amino benzosultam $\mathbf{4 m}$ was followed by insitu hydrazone formation, and finally $\mathrm{Zn}$-mediated reduction. In this way N-H derivative 8 was obtained in $68 \%$ yield for the 2-step procedure. It should be noted that the deprotection $/ \mathrm{N}-\mathrm{N}$ bond cleavage process also resulted in reduction of the $\mathrm{C}-\mathrm{C}$ double bond.
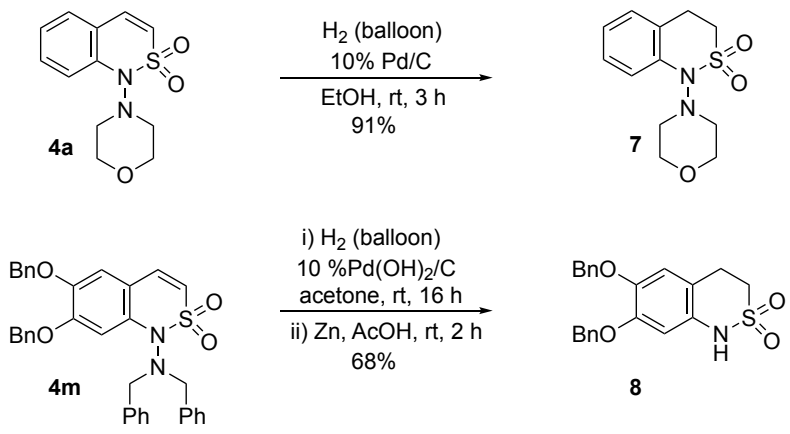

Scheme 5. C-C Double-bond reduction and the preparation of N$\mathrm{H}$ sultam-derivative 8 .

\section{Conclusions}

Although a single-step amino-sulfonylation/cyclisation sequence was not possible, the use of two discrete palladiumcatalysed processes, first an aminosulfonylation using $\mathrm{N}, \mathrm{N}-$ dialkylhydrazines as the $N$-nucleophiles, and DABSO as the $\mathrm{SO}_{2}$ source, and then a palladium-catalysed intramolecular sulfamidation, allowed a new entry into benzosultams to be achieved. Both steps use a catalyst generated from the combination of $\mathrm{Pd}(\mathrm{OAc})_{2} /$ CataCXium $\mathrm{A}$. The method tolerates good variation of the halide precursor, as well as variation of the hydrazine coupling partner. Access to the N-H benzosultam is possible if dibenzyl hydrazine is used as the coupling partner.

\section{Experimental}

\subsection{General information}

${ }^{1} \mathrm{H}$ and ${ }^{13} \mathrm{C}$ NMR spectra were obtained on a Brüker AVIII400 (400 MHz) or AVII (500 MHz) spectrometer. Proton-decoupled spectra are denoted as $\left\{{ }^{1} \mathrm{H}\right\}$. Chemical shifts $(\delta)$ were reported in parts per million (ppm) using the residual solvent signal as an internal standard $\left(\mathrm{CDCl}_{3}: \delta_{\mathrm{H}}=7.26 \mathrm{ppm}, \delta_{\mathrm{C}}=77.16 \mathrm{ppm}\right)$. All coupling constants ( $J$ values) were reported in Hertz $(\mathrm{Hz})$. Multiplicities were reported as follows: s, singlet; $d$, doublet; $t$, triplet; q, quartet; quin., quintet; m, multiplet. High-resolution mass spectrometry (HRMS) measurements were recorded on a Bruker Daltronics microTOF (ESI) spectrometer. Infrared spectra were recorded as thin films on a Bruker Tensor 27 FT-IR spectrometer. Flash chromatography was carried out using matrix 60 silica. All alkynes were distilled prior to use. The alkenyl iodide substrates were prepared from the corresponding aldehydes using Wittig chemistry, as previously reported.

\subsection{Synthesis of alkenylsulfonamides $\mathbf{3}$}

\subsubsection{General procedure A for the preparation of alkenylsulfonamides 3.}

An oven-dried tube was evacuated and backfilled with $\mathrm{N}_{2}$. It was then charged with the appropriate alkenyl iodide ( 1 equiv.), CataCXium A (20 mol\%), palladium(II) acetate (10 mol\%), TBAI ( 1 equiv.) and DABSO (1.1 equiv.). The solid reagents were weighed out in the air. The tube was then evacuated and backfilled with $\mathrm{N}_{2}$. The appropriate dialkyl hydrazine (1.5 equiv.) and 1,4-dioxane $[0.15 \mathrm{M}]$ were added via microsyringe through a subaseal under $\mathrm{N}_{2}$. The reaction mixture was stirred at $70{ }^{\circ} \mathrm{C}$ for the specified length of time. After cooling to RT, the suspension was filtered through a short pad of Celite and the residue washed sequentially with $\mathrm{CH}_{2} \mathrm{Cl}_{2}\left(20 \mathrm{~mL} \mathrm{mmol}^{-1}\right)$ and ether $(20 \mathrm{~mL}$ $\mathrm{mmol}^{-1}$ ) before being concentrated in vacuo. Purification via column chromatography yielded the corresponding alkenyl $\mathrm{N}$-aminosulfonamide.

\subsection{2. (Z)-2-(2-Bromophenyl)-N-(morpholin-4- $y$ l)ethenesulfonamide (3a)}

Prepared according to the general procedure A using (Z)-1bromo-2-(2-iodovinyl)benzene (74 mg, $0.24 \mathrm{mmol}, Z: E,>20: 1)$. The reaction mixture was stirred at $70{ }^{\circ} \mathrm{C}$ for $3.5 \mathrm{~h}$. Column chromatography (eluent: 7:3, ether:petrol) yielded alkenyl $\mathrm{N}$-aminosulfonamide 3a as an off-white crystalline solid (63 mg, 75\%, Z:E, 12:1): $\mathrm{mp} \mathrm{89-92}{ }^{\circ} \mathrm{C}\left(\mathrm{CH}_{2} \mathrm{Cl}_{2}\right) ; v_{\max }$ (neat) $/ \mathrm{cm}^{-1} 3202$, 3061, 2960, 2922, 2853, 1463, 1434, 1387, 1363, 1334, 1277, $1263,1220,1150,1110,1071,1043,1025 ; \delta_{\mathrm{H}}\left(400 \mathrm{MHz}, \mathrm{CDCl}_{3}\right)$ $7.89(1 \mathrm{H}, \mathrm{dd}, J$ 7.5, 1.5, $\operatorname{Ar} H), 7.61(1 \mathrm{H}, \mathrm{dd}, J 7.5,1.5, \operatorname{Ar} H)$, 7.41-7.31 (1H, app td, J 7.5, 1.5, ArH), 7.31-7.21 (1H, m, ArH), $7.23(1 \mathrm{H}, \mathrm{d}, J$ 12.0, $\mathrm{ArCH}=\mathrm{CHS}), 6.57(1 \mathrm{H}, \mathrm{d}, J 12.0$, $\mathrm{ArCH}=\mathrm{CHS}), 5.33(1 \mathrm{H}, \mathrm{s}, \mathrm{NH}), 3.73\left(4 \mathrm{H}, \mathrm{t}, J 4.5, \mathrm{~N}\left(\mathrm{CH}_{2} \mathrm{CH}_{2}\right)_{2} \mathrm{O}\right)$, $2.81\left(4 \mathrm{H}, \mathrm{t}, J 4.5, \mathrm{~N}\left(\mathrm{CH}_{2} \mathrm{CH}_{2}\right)_{2} \mathrm{O}\right) ; \delta_{\mathrm{C}}\left(101 \mathrm{MHz}, \mathrm{CDCl}_{3}\right) 140.3$ $(\mathrm{CH}), 133.1(\mathrm{C}), 132.3(\mathrm{CH}), 132.0(\mathrm{CH}), 131.0(\mathrm{CH}), 128.4(\mathrm{CH})$, $126.9(\mathrm{CH}), 123.4(\mathrm{C}), 66.6\left(2 \times \mathrm{CH}_{2}\right), 57.2\left(2 \times \mathrm{CH}_{2}\right) ; \mathrm{m} / z\left(\mathrm{ESI}^{+}\right)$ $719\left([2 \mathrm{M}+\mathrm{Na}]^{+}, 60 \%\right), 717\left([2 \mathrm{M}+\mathrm{Na}]^{+}, 100 \%\right), 715([2 \mathrm{M}+$ $\left.\mathrm{Na}]^{+}, 50 \%\right) ; m / z\left(\mathrm{ESI}^{-}\right) 383\left([\mathrm{M}+\mathrm{Cl}]^{-}, 100 \%\right), 381\left([\mathrm{M}+\mathrm{Cl}]^{-}\right.$, $77 \%)$; HRMS $\left(\mathrm{ESI}^{+}\right) \mathrm{C}_{12} \mathrm{H}_{15} \mathrm{BrN}_{2} \mathrm{NaO}_{3} \mathrm{~S}^{+}\left([\mathrm{M}+\mathrm{Na}]^{+}\right)$requires 370.9859 and 368.9879 , found 370.9855 and 368.9869 .

\subsection{3. (Z)-2-(2-Bromo-5-(trifluoromethyl)phenyl)- $N$ - morpholinoethenesulfonamide (3b)}

Prepared according to the general procedure using A (Z)-1bromo-2-(2-iodovinyl)-4-(trifluoromethyl)benzene (90 mg, 0.24 mmol, $Z: E,>20: 1)$. The reaction mixture was stirred at $70^{\circ} \mathrm{C}$ for 4 h. Column chromatography (eluent: 7:3, ether:petrol) yielded alkenyl $\mathrm{N}$-aminosulfonamide $\mathbf{3 b}$ as a white crystalline solid (79

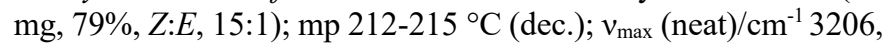
2962, 2926, 2856, 1603, 1459, 1414, 1327, 1262, 1206, 1155, $1128,1112,1081,1029 ; \delta_{\mathrm{H}}\left(400 \mathrm{MHz}, \mathrm{CDCl}_{3}\right) 8.15(1 \mathrm{H}, \mathrm{d}, J 1.5$, $\mathrm{Ar} H), 7.72(1 \mathrm{H}, \mathrm{d}, J 8.5, \mathrm{Ar} H), 7.49$ (1H, dd, $J 8.5,1.5, \mathrm{Ar} H), 7.21$ $(1 \mathrm{H}, \mathrm{d}, J 12.0, \mathrm{ArCH}=\mathrm{CHS}), 6.54(1 \mathrm{H}, \mathrm{d}, J 12.0, \mathrm{ArCH}=\mathrm{CHS})$, $5.34(1 \mathrm{H}$, br. s, $\mathrm{NH}), 3.75\left(4 \mathrm{H}, \mathrm{t}, J 4.5, \mathrm{~N}\left(\mathrm{CH}_{2} \mathrm{CH}_{2}\right)_{2} \mathrm{O}\right), 2.87(4 \mathrm{H}$, br. t, $\left.J 4.5, \mathrm{~N}\left(\mathrm{CH}_{2} \mathrm{CH}_{2}\right)_{2} \mathrm{O}\right) ; \delta_{\mathrm{C}}\left(126 \mathrm{MHz}, \mathrm{CDCl}_{3}\right) 139.5(\mathrm{CH})$, $134.0(\mathrm{C}), 132.8(\mathrm{CH}), 129.4(3)\left(\mathrm{C}, \mathrm{q}, J_{\mathrm{CF}} 32.5\right), 129.3(8)(\mathrm{CH})$, 
$129.2\left(\mathrm{CH}, \mathrm{q}, J_{\mathrm{CF}} 3.5\right), 127.3\left(\mathrm{CH}, \mathrm{q}, J_{\mathrm{CF}} 4.0\right), 127.0(\mathrm{C}, \mathrm{m}), 123.8$ $\left(\mathrm{C}, \mathrm{q}, J_{\mathrm{CF}} 272.5\right), 66.6\left(2 \times \mathrm{CH}_{2}\right), 57.6\left(2 \times \mathrm{CH}_{2}\right) ; \delta_{\mathrm{F}}(377 \mathrm{MHz}$, $\left.\mathrm{CDCl}_{3}\right)-62.4\left(\mathrm{~s}, \mathrm{ArCF}_{3}\right)\left\{{ }^{1} \mathrm{H}\right\} ; m / z\left(\mathrm{ESI}^{+}\right) 439\left([\mathrm{M}+\mathrm{Na}]^{+}, 100 \%\right)$, $437\left([\mathrm{M}+\mathrm{Na}]^{+}, 95 \%\right) ; \mathrm{HRMS}\left(\mathrm{ESI}^{+}\right) \mathrm{C}_{13} \mathrm{H}_{14} \mathrm{BrF}_{3} \mathrm{~N}_{2} \mathrm{NaO}_{3} \mathrm{~S}^{+}([\mathrm{M}+$ $\mathrm{Na}]^{+}$) requires 438.9732 and 436.9753 ; found 438.9745 and 436.9761.

\subsection{4. (Z)-2-(2-Bromo-4-fluorophenyl)- $\mathrm{N}$ - morpholinoethenesulfonamide (3c)}

Prepared according to the general procedure A using 2-bromo4-fluoro-1-(2-iodovinyl)benzene (78 mg, $0.24 \mathrm{mmol}, Z: E, 20: 1)$. The reaction mixture was stirred at $70{ }^{\circ} \mathrm{C}$ for $3.5 \mathrm{~h}$. Column chromatography (eluent: 7:3, ether:petrol) yielded alkenyl $\mathrm{N}$-aminosulfonamide $\mathbf{3 c}$ as a white crystalline solid $(61 \mathrm{mg}, 70 \%$, $Z: E, 20: 1) ; \mathrm{mp} 116-118^{\circ} \mathrm{C}\left(\mathrm{CH}_{2} \mathrm{Cl}_{2}\right) ; v_{\max }$ (neat) $/ \mathrm{cm}^{-1} 3191,3048$, 2978, 2923, 2855, 1622, 1591, 1477, 1457, 1390, 1357, 1315, $1265,1239,1189,1158,1140,1114,1073,1033 ; \delta_{\mathrm{H}}(400 \mathrm{MHz}$, $\left.\mathrm{CDCl}_{3}\right) 7.92(1 \mathrm{H}, \mathrm{dd}, J$ 9.0, 6.0, $\mathrm{Ar} H), 7.35(1 \mathrm{H}, \mathrm{dd}, J$ 8.0, 3.0, $\operatorname{Ar} H), 7.16(1 \mathrm{H}, \mathrm{d}, J 12.0, \operatorname{ArCH}=\mathrm{CHS}), 7.07(1 \mathrm{H}$, app td, $J 8.5$, 2.5, $\mathrm{Ar} H), 6.53(1 \mathrm{H}, \mathrm{d}, J 12.0, \mathrm{ArCH}=\mathrm{CHS}), 5.35(1 \mathrm{H}, \mathrm{s}, \mathrm{NH})$, $3.72\left(4 \mathrm{H}, \mathrm{t}, J 4.5, \mathrm{~N}\left(\mathrm{CH}_{2} \mathrm{CH}_{2}\right)_{2} \mathrm{O}\right), 2.83(4 \mathrm{H}$, br t, $J 4.5$, $\left.\mathrm{N}\left(\mathrm{CH}_{2} \mathrm{CH}_{2}\right)_{2} \mathrm{O}\right) ; \delta_{\mathrm{C}}\left(126 \mathrm{MHz}, \mathrm{CDCl}_{3}\right) 163.0\left(\mathrm{C}, \mathrm{d}, J_{\mathrm{CF}} 255.0\right)$, $139.4(\mathrm{CH}), 133.6\left(\mathrm{CH}, \mathrm{d}, J_{\mathrm{CF}} 9.0\right), 129.3\left(\mathrm{C}, \mathrm{d}, J_{\mathrm{CF}} 4.0\right), 128.5$ $(\mathrm{CH}), 124.0\left(\mathrm{C}, \mathrm{d}, J_{\mathrm{CF}} 10.0\right), 119.8\left(\mathrm{CH}, \mathrm{d}, J_{\mathrm{CF}} 24.5\right), 114.5(\mathrm{CH}$, $\left.\mathrm{d}, J_{\mathrm{CF}} 21.5\right), 66.6\left(2 \times \mathrm{CH}_{2}\right), 57.4\left(2 \times \mathrm{CH}_{2}\right) ; \delta_{\mathrm{F}}\left(377 \mathrm{MHz}, \mathrm{CDCl}_{3}\right)$ $-108.8(\mathrm{~s}, \mathrm{Ar} F)\left\{{ }^{1} \mathrm{H}\right\} ; m / z\left(\mathrm{ESI}^{-}\right) 365\left([\mathrm{M}-\mathrm{H}]^{-}, 90 \%\right), 363$ ([M $\left.\mathrm{H}]^{-}, 100 \%\right)$; HRMS $\left(\mathrm{ESI}^{+}\right) \mathrm{C}_{12} \mathrm{H}_{14} \mathrm{BrFN}_{2} \mathrm{NaO}_{3} \mathrm{~S}^{+}\left([\mathrm{M}+\mathrm{Na}]^{+}\right)$ requires 388.9764 and 386.9785 , found 388.9771 and 386.9792 .

\subsection{5. (Z)-2-(2-Bromo-5-fluorophenyl)- $N$ - morpholinoethenesulfonamide (3d)}

Prepared according to the general procedure A using $(Z)-1$ bromo-4-fluoro-2-(2-iodovinyl)benzene ( $78 \mathrm{mg}, 0.24 \mathrm{mmol}, Z: E$, 18:1). The reaction mixture was stirred at $70{ }^{\circ} \mathrm{C}$ for $3.5 \mathrm{~h}$. Column chromatography (eluent: 7:3, ether:petrol) yielded alkenyl $\mathrm{N}$-aminosulfonamide 3d as a white crystalline solid (57 mg, 65\%, $Z: E, 15: 1) ; \mathrm{mp} 125-127^{\circ} \mathrm{C}\left(\mathrm{CH}_{2} \mathrm{Cl}_{2}\right) ; v_{\max }$ (neat) $/ \mathrm{cm}^{-1} 3135,2867$, $1575,1460,1340,1267,1200,1156,1104,1069,1033 ; \delta_{\mathrm{H}}(500$ $\left.\mathrm{MHz}, \mathrm{CDCl}_{3}\right) 7.78(1 \mathrm{H}, \mathrm{dd}, J$ 9.0, 3.0, $\mathrm{ArH}), 7.53(1 \mathrm{H}, \mathrm{dd}, J 9.0$, 5.0, $\mathrm{ArH}), 7.16(1 \mathrm{H}, \mathrm{d}, J 12.0, \mathrm{ArCH}=\mathrm{CHS}), 7.00-6.95(1 \mathrm{H}, \mathrm{m}$, $\mathrm{ArH}), 6.52(1 \mathrm{H}, \mathrm{d}, J 12.0, \mathrm{ArCH}=\mathrm{CHS}), 5.36(1 \mathrm{H}, \mathrm{s}, \mathrm{NH}), 3.75$ $\left(4 \mathrm{H}, \quad \mathrm{t}, J 4.5, \quad \mathrm{~N}\left(\mathrm{CH}_{2} \mathrm{CH}_{2}\right)_{2} \mathrm{O}\right), 2.86(4 \mathrm{H}, \quad$ br. $\mathrm{t}, J 4.5$, $\left.\mathrm{N}\left(\mathrm{CH}_{2} \mathrm{CH}_{2}\right)_{2} \mathrm{O}\right) ; \delta_{\mathrm{C}}\left(126 \mathrm{MHz}, \mathrm{CDCl}_{3}\right) 161.2\left(\mathrm{C}, \mathrm{d}, J_{\mathrm{CF}} 247.0\right)$, $139.6\left(\mathrm{CH}, \mathrm{d}, J_{\mathrm{CF}} 2.0\right), 134.7\left(\mathrm{C}, \mathrm{d}, J_{\mathrm{CF}} 9.0\right), 133.5\left(\mathrm{CH}, \mathrm{d}, J_{\mathrm{CF}} 7.5\right)$, $129.0(\mathrm{CH}), 119.5\left(\mathrm{CH}, \mathrm{d}, J_{\mathrm{CF}} 24.5\right), 118.3\left(\mathrm{CH}, \mathrm{d}, J_{\mathrm{CF}} 23.0\right), 117.7$ $\left(\mathrm{C}, \mathrm{d}, J_{\mathrm{CF}} 3.5\right), 66.7\left(2 \times \mathrm{CH}_{2}\right), 57.5\left(2 \times \mathrm{CH}_{2}\right) ; \delta_{\mathrm{F}}(377 \mathrm{MHz}$, $\left.\mathrm{CDCl}_{3}\right)-114.5(\mathrm{~s}, \mathrm{Ar} F)\left\{{ }^{1} \mathrm{H}\right\} ; \mathrm{m} / z\left(\mathrm{ESI}^{-}\right) 365\left([\mathrm{M}-\mathrm{H}]^{-}, 95 \%\right), 363$ ([M - H]', 100\%); HRMS (ESI-) $\left.\mathrm{C}_{12} \mathrm{H}_{13} \mathrm{BrFN}_{2} \mathrm{O}_{3} \mathrm{~S}^{-}\left([\mathrm{M}-\mathrm{H}]^{-}\right]\right)$ requires 364.9801 and 362.9825 , found 364.9799 and 362.9820).

\subsection{6. (Z)-2-(1-Bromonaphthalen-2-yl)-N- morpholinoethenesulfonamide (3e)}

Prepared according to the general procedure A using (Z)-1bromo-2-(2-iodovinyl)naphthalene $(86 \mathrm{mg}, 0.24 \mathrm{mmol}, Z: E$, $>20: 1)$. The reaction mixture was stirred at $70^{\circ} \mathrm{C}$ for $4 \mathrm{~h}$. Column chromatography (eluent: 7:3, ether:petrol) yielded alkenyl $\mathrm{N}$ aminosulfonamide $\mathbf{3 e}$ as a white crystalline solid $(72 \mathrm{mg}, 75 \%$, $Z: E, 15: 1) ; \mathrm{mp} 152-156^{\circ} \mathrm{C}\left(\mathrm{CH}_{2} \mathrm{Cl}_{2}\right), v_{\max }$ (neat) $/ \mathrm{cm}^{-1} 3191,3041$, 2959, 2926, 2857, 1632, 1553, 1498, 1457, 1417, 1386, 1364, $1320,1270,1238,1142,1105,1072,1016 ; \delta_{\mathrm{H}}(400 \mathrm{MHz}$, $\left.\left(\mathrm{CD}_{3}\right)_{2} \mathrm{SO}\right) 8.88(1 \mathrm{H}, \mathrm{s}, \mathrm{N} H), 8.23(1 \mathrm{H}, \mathrm{d}, J 8.5, \mathrm{Ar} H), 8.03-7.90$ $(3 \mathrm{H}, \mathrm{m}, 3 \times \mathrm{Ar} H), 7.71(1 \mathrm{H}$, app t, $J$ 7.0 $\mathrm{ArH})$ overlapping 7.65
(1H, app t, $J$ 8.0, $\mathrm{ArH}), 7.43(1 \mathrm{H}, \mathrm{d}, J 11.5, \mathrm{ArCH}=\mathrm{CHS}), 6.75$ $(1 \mathrm{H}, \mathrm{d}, J 11.5, \mathrm{ArCH}=\mathrm{CHS}), 3.64\left(4 \mathrm{H}\right.$, br. t, $\left.J 4.0, \mathrm{~N}\left(\mathrm{CH}_{2} \mathrm{CH}_{2}\right)_{2} \mathrm{O}\right)$, 2.84-2.78 (4H, m, N $\left.\left(\mathrm{CH}_{2} \mathrm{CH}_{2}\right)_{2} \mathrm{O}\right) ; \delta_{\mathrm{C}}\left(126 \mathrm{MHz}, \mathrm{CDCl}_{3}\right) 141.5$ $(\mathrm{CH}), 134.6(\mathrm{C}), 131.8(9)(\mathrm{C}), 131.7(7)(\mathrm{C}), 128.4(\mathrm{CH}), 128.3(3)$ $(\mathrm{CH}), 128.2(6)(\mathrm{CH}), 127.9(\mathrm{CH}), 127.7(\mathrm{CH}), 127.3(\mathrm{CH}), 127.1$ $(\mathrm{CH}), 124.2(\mathrm{C}), 66.8\left(2 \times \mathrm{CH}_{2}\right), 57.4\left(2 \times \mathrm{CH}_{2}\right) ; \mathrm{m} / z\left(\mathrm{ESI}^{-}\right) 397$ $\left([\mathrm{M}-\mathrm{H}]^{-}, 100 \%\right), 395\left([\mathrm{M}-\mathrm{H}]^{-}, 80 \%\right)$; HRMS $\left(\mathrm{ESI}^{+}\right)$ $\mathrm{C}_{16} \mathrm{H}_{17} \mathrm{BrN}_{2} \mathrm{NaO}_{3} \mathrm{~S}^{+}\left([\mathrm{M}+\mathrm{Na}]^{+}\right)$requires 421.0015 and 419.0035, found 421.0019 and 419.0042 .

\subsection{7. (Z)-2-(6-Bromobenzo[d][1,3]dioxol-5-yl)- $N$ - morpholinoethenesulfonamide (3f)}

Prepared according to the general procedure A using $(Z)$ bromo-6-(2-iodovinyl)benzo[ $d][1,3]$ dioxole $(85 \mathrm{mg}, 0.24 \mathrm{mmol}$, $Z: E,>20: 1)$. The reaction mixture was stirred at $70{ }^{\circ} \mathrm{C}$ for $3 \mathrm{~h}$. Column chromatography (eluent: 7:3, ether:petrol) yielded alkenyl $N$-aminosulfonamide $\mathbf{3 f}$ as a white crystalline solid (73 mg, $78 \%$, $Z: E, 20: 1) ; \mathrm{mp} 128-131{ }^{\circ} \mathrm{C}\left(\mathrm{CH}_{2} \mathrm{Cl}_{2}\right) ; v_{\max }$ (neat) $/ \mathrm{cm}^{-1} 3157,3044$, 2958, 2931, 2852, 1621, 1502, 1275, 1415, 1388, 1363, 1318, $1260,1246,1197,1142,1106,1070,1036 ; \delta_{\mathrm{H}}\left(400 \mathrm{MHz}, \mathrm{CDCl}_{3}\right)$ $7.67(1 \mathrm{H}, \mathrm{s}, \mathrm{ArH}), 7.15(1 \mathrm{H}, \mathrm{d}, J 12.0, \mathrm{ArCH}=\mathrm{CHS}), 7.04(1 \mathrm{H}, \mathrm{s}$, $\mathrm{ArH}), 6.40(1 \mathrm{H}, \mathrm{d}, J 12.0, \mathrm{ArCH}=\mathrm{CHS}), 6.03\left(2 \mathrm{H}, \mathrm{s}, \mathrm{OCH}_{2} \mathrm{O}\right)$, $5.58(1 \mathrm{H}, \mathrm{s}, \mathrm{NH}), 3.74\left(4 \mathrm{H}, \mathrm{t}, J 4.5, \mathrm{~N}\left(\mathrm{CH}_{2} \mathrm{CH}_{2}\right)_{2} \mathrm{O}\right), 2.86(4 \mathrm{H}$, br. $\left.\mathrm{t}, J 4.5, \mathrm{~N}\left(\mathrm{CH}_{2} \mathrm{CH}_{2}\right)_{2} \mathrm{O}\right) ; \delta_{\mathrm{C}}\left(101 \mathrm{MHz}, \mathrm{CDCl}_{3}\right) 149.8(\mathrm{C}), 147.0$ (C), $140.5(\mathrm{CH}), 126.9(\mathrm{CH}), 125.9(\mathrm{C}), 116.1(\mathrm{C}), 112.4(\mathrm{CH})$, $112.0(\mathrm{CH}), 102.3(\mathrm{CH}), 66.7\left(2 \times \mathrm{CH}_{2}\right), 57.3\left(2 \times \mathrm{CH}_{2}\right) ; \mathrm{m} / z\left(\mathrm{ESI}^{-}\right.$ ) $391\left([\mathrm{M}-\mathrm{H}]^{-}, 100 \%\right), 389\left([\mathrm{M}-\mathrm{H}]^{-}, 98 \%\right)$; HRMS (ESI $\left.{ }^{-}\right)$ $\mathrm{C}_{13} \mathrm{H}_{14} \mathrm{BrN}_{2} \mathrm{O}_{5} \mathrm{~S}^{-}\left([\mathrm{M}-\mathrm{H}]^{-}\right)$requires 390.9792 and 388.9812, found 390.9793 and 388.9819 .

\subsection{8. (Z)-2-(5-(Benzyloxy)-2-bromophenyl)- $N$ - morpholinoethenesulfonamide (3g)}

Prepared according to the general procedure A using 4(benzyloxy)-1-bromo-2-(2-iodovinyl)benzene (100 mg, 0.24 mmol, $Z: E, 15: 1)$. The reaction mixture was stirred at $70{ }^{\circ} \mathrm{C}$ for 4 h. Column chromatography (eluent: 7:3, ether:petrol) yielded alkenyl $N$-aminosulfonamide $\mathbf{3 g}$ as white needles $(86 \mathrm{mg}, 79 \%$, 8:1); mp $178-180^{\circ} \mathrm{C}\left(\mathrm{CH}_{2} \mathrm{Cl}_{2}\right) ; v_{\max }$ (neat) $/ \mathrm{cm}^{-1} 3207,3059,2952$, 2855, 2820, 1616, 1589, 1460, 1446, 1404, 1363, 1333, 1262, $1249,1231,1179,1156,1112,1046,1016 ; \delta_{\mathrm{H}}\left(400 \mathrm{MHz}, \mathrm{CDCl}_{3}\right)$ $7.61(1 \mathrm{H}, \mathrm{d}, J 3.0, \operatorname{Ar} H), 7.46(1 \mathrm{H}, \mathrm{d}, J 9.0, \mathrm{Ar} H), 7.44-7.32(5 \mathrm{H}$, $\mathrm{m}, 5 \times \mathrm{OBn} H), 7.16(1 \mathrm{H}, \mathrm{d}, J 12.0, \mathrm{ArCH}=\mathrm{CHS}), 6.90(1 \mathrm{H}, \mathrm{dd}, J$ 9.0, 3.0, $\mathrm{ArH}), 6.54(1 \mathrm{H}, \mathrm{d}, J 12.0, \mathrm{ArCH}=\mathrm{CHS}), 5.11(2 \mathrm{H}, \mathrm{s}$, $\left.\mathrm{OCH}_{2} \mathrm{Ph}\right), 4.99(1 \mathrm{H}, \mathrm{s}, \mathrm{NH}), 3.67\left(4 \mathrm{H}, \mathrm{t}, J 4.5, \mathrm{~N}\left(\mathrm{CH}_{2} \mathrm{CH}_{2}\right)_{2} \mathrm{O}\right)$, $2.68\left(4 \mathrm{H}\right.$, br. t, $\left.J 4.5, \mathrm{~N}\left(\mathrm{CH}_{2} \mathrm{CH}_{2}\right)_{2} \mathrm{O}\right) ; \delta_{\mathrm{C}}\left(101 \mathrm{MHz}, \mathrm{CDCl}_{3}\right) 157.5$ (C), $140.1(\mathrm{CH}), 136.6(\mathrm{CH}), 133.6(4)(\mathrm{CH}), 133.1(\mathrm{CH}), 129.0$ (C), $128.8(2 \times \mathrm{CH}), 128.2(\mathrm{C}), 127.6(4)(2 \times \mathrm{CH}), 118.9(\mathrm{CH})$, 118.1 (CH), $114.5(\mathrm{C}), 70.3\left(\mathrm{CH}_{2}\right), 66.6\left(2 \times \mathrm{CH}_{2}\right), 57.2\left(2 \times \mathrm{CH}_{2}\right)$; $m / z\left(\mathrm{ESI}^{+}\right) 477\left([\mathrm{M}+\mathrm{Na}]^{+}, 95 \%\right) 475\left([\mathrm{M}+\mathrm{Na}]^{+}, 100 \%\right) ; \mathrm{HRMS}$ $\left(\mathrm{ESI}^{+}\right) \mathrm{C}_{19} \mathrm{H}_{21} \mathrm{BrN}_{2} \mathrm{NaO}_{4} \mathrm{~S}^{+}\left([\mathrm{M}+\mathrm{Na}]^{+}\right)$requires 477.0278 and 475.0298, found 477.0287 and 475.0306; Anal. Calcd. (\%) for $\mathrm{C}_{19} \mathrm{H}_{21} \mathrm{BrN}_{2} \mathrm{O}_{4} \mathrm{~S}: \mathrm{C}$ 50.34, H 4.67, N 6.18; found C 50.06, H 4.52, N 6.03 .

\subsection{9. (Z)-2-(2-Bromo-4-methylphenyl)-N- morpholinoethenesulfonamide $(\mathbf{3 h})$}

Prepared according to the general procedure A using 2-bromo1-(2-iodovinyl)-4-methylbenzene (78 mg, $0.24 \mathrm{mmol}, Z: E, 18: 1)$. The reaction mixture was stirred at $70{ }^{\circ} \mathrm{C}$ for $3.5 \mathrm{~h}$. Column chromatography (eluent: 7:3, ether:petrol) yielded alkenyl $\mathrm{N}$-aminosulfonamide $\mathbf{3 h}$ as a white crystalline solid $(68 \mathrm{mg}, 78 \%$, $Z: E, 15: 1) ; \mathrm{mp} 148-149^{\circ} \mathrm{C}\left(\mathrm{CH}_{2} \mathrm{Cl}_{2}\right) ; v_{\max }$ (neat) $/ \mathrm{cm}^{-1} 3245,2852$, 
$1619,1600,1446,1360,1330,1283,1267,1152,1106,1071$, $1042 ; \delta_{\mathrm{H}}\left(500 \mathrm{MHz}, \mathrm{CDCl}_{3}\right) 7.81(1 \mathrm{H}, \mathrm{d}, J 8.0, \mathrm{ArH}), 7.43(1 \mathrm{H}, \mathrm{d}$, $J$ 1.0, $\mathrm{Ar} H), 7.20(1 \mathrm{H}, \mathrm{d}, J 12.0, \mathrm{ArCH}=\mathrm{CHS}), 7.16-7.13(1 \mathrm{H}, \mathrm{m}$, $\mathrm{ArH}), 6.51(1 \mathrm{H}, \mathrm{d}, J 12.0, \mathrm{ArCH}=\mathrm{CHS}), 5.31(1 \mathrm{H}, \mathrm{s}, \mathrm{NH}), 3.71$ $\left(4 \mathrm{H}, \mathrm{t}, J 4.5, \mathrm{~N}\left(\mathrm{CH}_{2} \mathrm{CH}_{2}\right)_{2} \mathrm{O}\right), 2.80\left(4 \mathrm{H}\right.$, br. t, $\left.J 4.5, \mathrm{~N}\left(\mathrm{CH}_{2} \mathrm{CH}_{2}\right)_{2} \mathrm{O}\right)$, $2.35(3 \mathrm{H}, \mathrm{s}, \mathrm{ArMe}) ; \delta_{\mathrm{C}}\left(126 \mathrm{MHz}, \mathrm{CDCl}_{3}\right) 141.9(\mathrm{CH}), 140.4(\mathrm{CH})$, $132.9(\mathrm{CH}), 131.9(\mathrm{CH}), 130.1(\mathrm{C}), 128.0(2 \times \mathrm{CH}), 123.6(\mathrm{C})$, $66.7\left(2 \times \mathrm{CH}_{2}\right), 57.3\left(2 \times \mathrm{CH}_{2}\right), 21.2\left(\mathrm{CH}_{3}\right) ; \mathrm{m} / z\left(\mathrm{ESI}^{-}\right) 361([\mathrm{M}-$ $\left.\mathrm{H}]^{-}, \quad 95 \%\right), \quad 359 \quad\left([\mathrm{M}-\mathrm{H}]^{-}, \quad 100 \%\right) ; \quad \mathrm{HRMS} \quad\left(\mathrm{ESI}^{+}\right)$ $\mathrm{C}_{13} \mathrm{H}_{17} \mathrm{BrN}_{2} \mathrm{NaO}_{3} \mathrm{~S}^{+}\left([\mathrm{M}+\mathrm{Na}]^{+}\right)$requires 385.0015 and 383.0035, found 385.0026 and 383.0048; Anal. Calcd. (\%) for $\mathrm{C}_{13} \mathrm{H}_{17} \mathrm{BrN}_{2} \mathrm{O}_{3} \mathrm{~S}$ : C 43.22, H 4.74, N 7.75; found C 43.01, H 4.81, N 7.66 .

\subsubsection{0. (Z)-2-(2-Bromo-4,5-dimethoxyphenyl)- $N$ - morpholinoethenesulfonamide (3i)}

Prepared according to the general procedure A using 1-bromo2-(2-iodovinyl)-4,5-dimethoxybenzene ( $89 \mathrm{mg}, 0.24 \mathrm{mmol}, Z: E$, $>20: 1$ ). The reaction mixture was stirred at $70{ }^{\circ} \mathrm{C}$ for $3 \mathrm{~h}$. Column chromatography (eluent: 7:3, ether:petrol) yielded alkenyl $\mathrm{N}$-aminosulfonamide $\mathbf{3 i}$ as a white crystalline solid $(82 \mathrm{mg}, 84 \%$, $Z: E, 15: 1) ; \mathrm{mp} 181-185^{\circ} \mathrm{C}\left(\mathrm{CH}_{2} \mathrm{Cl}_{2}\right) ; v_{\max }$ (neat) $/ \mathrm{cm}^{-1} 3217,3006$, 2973, 2932, 2858, 1596, 1500, 1463, 1442, 1392, 1325, 1308, $1267,1232,1203,1186,1157,1132,1114,1021 ; \delta_{\mathrm{H}}(400 \mathrm{MHz}$, $\left.\mathrm{CDCl}_{3}\right) 7.67(1 \mathrm{H}, \mathrm{s}, \mathrm{Ar} H), 7.17(1 \mathrm{H}, \mathrm{d}, J 12.0, \mathrm{ArCH}=\mathrm{CHS}), 7.05$ $(1 \mathrm{H}, \mathrm{s}, \mathrm{Ar} H), 6.51(1 \mathrm{H}, \mathrm{d}, J 12.0, \mathrm{ArCH}=\mathrm{CHS}), 5.19(1 \mathrm{H}, \mathrm{s}, \mathrm{NH})$, $3.91(3 \mathrm{H}, \mathrm{s}, \mathrm{OMe})$ overlapping $3.90(3 \mathrm{H}, \mathrm{s}, \mathrm{OMe}), 3.69(4 \mathrm{H}, \mathrm{t}, J$ 4.5, $\left.\mathrm{N}\left(\mathrm{CH}_{2} \mathrm{CH}_{2}\right)_{2} \mathrm{O}\right), 2.76\left(4 \mathrm{H}\right.$, br. t, $\left.J 4.5, \mathrm{~N}\left(\mathrm{CH}_{2} \mathrm{CH}_{2}\right)_{2} \mathrm{O}\right) ; \delta_{\mathrm{C}}$ $\left(126 \mathrm{MHz}, \mathrm{CDCl}_{3}\right) 150.9(\mathrm{C}), 148.0(\mathrm{C}), 140.0(\mathrm{CH}), 127.7(\mathrm{CH})$, $124.9(\mathrm{C}), 115.5(\mathrm{C}), 115.0(2 \times \mathrm{CH}), 66.7\left(2 \times \mathrm{CH}_{2}\right), 57.3(2 \times$ $\left.\mathrm{CH}_{2}\right), 56.5\left(\mathrm{CH}_{3}\right), 56.4\left(\mathrm{CH}_{3}\right) ; \mathrm{m} / z\left(\mathrm{ESI}^{+}\right) 431\left([\mathrm{M}+\mathrm{Na}]^{+}, 95 \%\right)$, $429\left([\mathrm{M}+\mathrm{Na}]^{+}, 100 \%\right) ; \mathrm{HRMS}\left(\mathrm{ESI}^{+}\right) \mathrm{C}_{14} \mathrm{H}_{19} \mathrm{BrN}_{2} \mathrm{NaO}_{5} \mathrm{~S}^{+}([\mathrm{M}+$ $\mathrm{Na}]^{+}$) requires 431.0070 and 429.0090 , found 431.0083 and 429.0104 .

\subsubsection{1. (Z)-2-(2-Bromothiophen-3-yl)- $\mathrm{N}$ - morpholinoethenesulfonamide (3j)}

Prepared according to the general procedure A using 2-bromo3-(2-iodovinyl)thiophene (76 mg, $0.24 \mathrm{mmol}, Z: E, 10: 1)$. The reaction mixture was stirred at $70{ }^{\circ} \mathrm{C}$ for $3 \mathrm{~h}$. Column chromatography (eluent: 7:3, ether:petrol) yielded alkenyl $\mathrm{N}$-aminosulfonamide $\mathbf{3 j}$ as a white crystalline solid (50 mg, 59\%, $Z: E, 10: 1) ; \mathrm{mp} 185-186{ }^{\circ} \mathrm{C}\left(\mathrm{CH}_{2} \mathrm{Cl}_{2}\right) ; v_{\max }$ (neat) $/ \mathrm{cm}^{-1} 3207,3108$, 2962, 2920, 2855, 1605, 1456, 1412, 1362, 1337, 1264, 1149, $1109 ; \delta_{\mathrm{H}}\left(400 \mathrm{MHz}, \mathrm{CDCl}_{3}\right) 7.60(1 \mathrm{H}, \mathrm{d}, J 5.5, \mathrm{CH}=\mathrm{CHS}), 7.31$ (1H, dd, $J$ 12.0, 1.0, $\mathrm{ArCH}=\mathrm{CHS}), 7.09(1 \mathrm{H}, \mathrm{d}, J 5.5, \mathrm{CH}=\mathrm{CHS})$, $6.32(1 \mathrm{H}, \mathrm{d}, J 12.0, \mathrm{ArCH}=\mathrm{CHS}), 5.53(1 \mathrm{H}, \mathrm{s}, \mathrm{N} H), 3.65(4 \mathrm{H}, \mathrm{t}, J$ 4.5, N( $\left.\left(\mathrm{CH}_{2} \mathrm{CH}_{2}\right)_{2} \mathrm{O}\right), 2.83\left(4 \mathrm{H}\right.$, br. t, $\left.J 4.5, \mathrm{~N}\left(\mathrm{CH}_{2} \mathrm{CH}_{2}\right)_{2} \mathrm{O}\right) ; \delta_{\mathrm{C}}(126$ $\left.\mathrm{MHz}, \mathrm{CDCl}_{3}\right) 132.7(\mathrm{CH}), 132.0(\mathrm{CH}), 130.0(\mathrm{CH}), 128.9(\mathrm{C})$, $123.2(\mathrm{CH}), 120.0(\mathrm{C}), 66.7\left(2 \times \mathrm{CH}_{2}\right), 57.2\left(2 \times \mathrm{CH}_{2}\right) ; \mathrm{m} / z\left(\mathrm{ESI}^{+}\right)$ $377\left([\mathrm{M}+\mathrm{Na}]^{+}, 90 \%\right), 375\left([\mathrm{M}+\mathrm{Na}]^{+}, 100 \%\right) ;$ HRMS $\left(\mathrm{ESI}^{+}\right)$ $\mathrm{C}_{10} \mathrm{H}_{13} \mathrm{BrN}_{2} \mathrm{NaO}_{3} \mathrm{~S}_{2}^{+}\left([\mathrm{M}+\mathrm{Na}]^{+}\right)$requires 376.9422 and 374.9443, found 376.9432 and 374.9455 .

\subsubsection{2. (Z)-2-(2-Bromopyridin-3-yl)- $N$ - morpholinoethenesulfonamide $(3 \mathbf{k})$}

Prepared according to the general procedure A using $(Z)-2$ bromo-3-(2-iodovinyl)pyridine (74 mg, $0.24 \mathrm{mmol}, Z: E,>20: 1$ ). The reaction mixture was stirred at $70{ }^{\circ} \mathrm{C}$ for $3 \mathrm{~h}$. Column chromatography (eluent: 7:3, ether:petrol) yielded alkenyl $\mathrm{N}$-aminosulfonamide 3k as a pale yellow crystalline solid (69 $\mathrm{mg}$, $83 \%, Z: E,>20: 1), \mathrm{mp} 118-121^{\circ} \mathrm{C}\left(\mathrm{CH}_{2} \mathrm{Cl}_{2}\right) ; v_{\max }$ (neat) $/ \mathrm{cm}^{-1} 3140$,
3031, 2978, 2887, 1618, 1572, 1555, 1460, 1396, 1370, 1337, $1279,1265,1206,1150,1123,1103,1070,1061,1051,1012 ; \delta_{\mathrm{H}}$ $\left(400 \mathrm{MHz}, \mathrm{CDCl}_{3}\right) 8.37(1 \mathrm{H}, \mathrm{dd}, J 5.0,1.5, \mathrm{ArH}), 8.21-8.13(1 \mathrm{H}$, $\mathrm{m}, \operatorname{Ar} H), 7.32(1 \mathrm{H}, \mathrm{dd}, J 7.5,5.0, \operatorname{Ar} H), 7.16(1 \mathrm{H}, \mathrm{d}, J 11.5$, $\mathrm{ArCH}=\mathrm{CHS}), 6.61(1 \mathrm{H}, \mathrm{d}, J 11.5, \mathrm{ArCH}=\mathrm{CHS}), 5.36(1 \mathrm{H}, \mathrm{s}, \mathrm{NH})$, $3.72\left(4 \mathrm{H}, \mathrm{t}, J 4.5, \mathrm{~N}\left(\mathrm{CH}_{2} \mathrm{CH}_{2}\right)_{2} \mathrm{O}\right), 2.85(4 \mathrm{H}$, br. t, $J 4.5$, $\left.\mathrm{N}\left(\mathrm{CH}_{2} \mathrm{CH}_{2}\right)_{2} \mathrm{O}\right) ; \delta_{\mathrm{C}}\left(101 \mathrm{MHz}, \mathrm{CDCl}_{3}\right) 138.3(\mathrm{C}), 138.1(\mathrm{CH})$, $135.1(\mathrm{CH}), 134.3(\mathrm{CH}), 133.5(\mathrm{CH}), 127.9(\mathrm{CH}), 120.2(\mathrm{C}), 66.6$ $\left(2 \times \mathrm{CH}_{2}\right), 56.7\left(2 \times \mathrm{CH}_{2}\right) ; \mathrm{m} / z\left(\mathrm{ESI}^{+}\right) 372\left([\mathrm{M}+\mathrm{Na}]^{+}, 100 \%\right), 370$ $\left([\mathrm{M}+\mathrm{Na}]^{+}, 95 \%\right) ; \mathrm{HRMS}\left(\mathrm{ESI}^{+}\right) \mathrm{C}_{11} \mathrm{H}_{14} \mathrm{BrN}_{3} \mathrm{NaO}_{3} \mathrm{~S}^{+}\left([\mathrm{M}+\mathrm{Na}]^{+}\right)$ requires 371.9811 and 369.9831 , found 371.9826 and 369.9849 .

\subsubsection{3. (Z)-2-(2-Bromo-4,5-dimethoxyphenyl)- $N-(4-$ methylpiperazin-1-yl)ethenesulfonamide (3l)}

Prepared according to the general procedure A using (Z)-1bromo-2-(2-iodovinyl)-4,5-dimethoxybenzene (89 $\mathrm{mg}, \quad 0.24$ mmol, $Z: E,>20: 1)$. The reaction mixture was stirred at $70{ }^{\circ} \mathrm{C}$ for $3 \mathrm{~h}$. Column chromatography (eluent: $4 \% \mathrm{MeOH}$ in $\mathrm{CH}_{2} \mathrm{Cl}_{2}$ ) yielded alkenyl $\mathrm{N}$-aminosulfonamide $\mathbf{3 l}$ as a white crystalline solid (75 mg, 74\%, Z:E, 11:1); mp $160{ }^{\circ} \mathrm{C}$ (dec) $\left(\mathrm{CH}_{2} \mathrm{Cl}_{2}\right) ; v_{\max }$ (neat) $/ \mathrm{cm}^{-1} 3227,2926,2851,1597,1502,1463,1439,1389,1326$, $1271,1209,1181,1150,1027 ; \delta_{\mathrm{H}}\left(400 \mathrm{MHz}, \mathrm{CDCl}_{3}\right) 7.63(1 \mathrm{H}, \mathrm{s}$, $\mathrm{Ar} H), 7.16(1 \mathrm{H}, \mathrm{d}, J 12.0, \mathrm{ArCH}=\mathrm{CHS}), 7.04(1 \mathrm{H}, \mathrm{s}, \mathrm{ArH}), 6.46$ $(1 \mathrm{H}, \mathrm{d}, J 12.0, \mathrm{ArCH}=\mathrm{CHS}), 6.15(1 \mathrm{H}$, br. s, NH), $3.91(3 \mathrm{H}, \mathrm{s}$, $\mathrm{OMe}$ ) overlapping $3.90(3 \mathrm{H}, \mathrm{s}, \mathrm{OMe}), 3.12(4 \mathrm{H}$, br. $\mathrm{s}$, $\left.\mathrm{N}\left(\mathrm{CH}_{2} \mathrm{CH}_{2}\right)_{2} \mathrm{NMe}\right)$ overlapping $3.03 \quad(4 \mathrm{H}$, br. s, $\left.\mathrm{N}\left(\mathrm{CH}_{2} \mathrm{CH}_{2}\right)_{2} \mathrm{NMe}\right), 2.64(3 \mathrm{H}, \mathrm{s}, \mathrm{NMe}) ; \delta_{\mathrm{C}}\left(126 \mathrm{MHz}, \mathrm{CDCl}_{3}, \mathrm{Z}\right.$ isomer) $151.1(\mathrm{C}), 148.1(\mathrm{C}), 140.3(\mathrm{CH}), 127.6(\mathrm{CH}), 124.8(\mathrm{C})$, $115.3(\mathrm{C}), 115.0(0)(\mathrm{CH}), 114.9(7)(\mathrm{CH}), 56.5\left(\mathrm{CH}_{3}\right), 56.4\left(\mathrm{CH}_{3}\right)$, $53.7\left(2 \times \mathrm{CH}_{2}\right), 43.9\left(2 \times \mathrm{CH}_{2}\right), 29.8\left(\mathrm{CH}_{3}\right) ; \mathrm{HRMS}\left(\mathrm{FI}^{+}\right)$ $\mathrm{C}_{15} \mathrm{H}_{22} \mathrm{BrN}_{3} \mathrm{NaO}_{4} \mathrm{~S}^{+}\left([\mathrm{M}]^{+}\right)$requires 422.0523 and 421.0495 , found 422.0348 and 421.0372 .

\subsubsection{4. (Z)- $N^{\prime}, N^{\prime}$-Dibenzyl-2-(2-bromo-4,5- dimethoxyphenyl)ethenesulfonohydrazide (3m)}

Prepared according to the general procedure A using (Z)-1bromo-2-(2-iodovinyl)-4,5-dimethoxybenzene (89 $\mathrm{mg}, \quad 0.24$ mmol, $Z: E,>20: 1)$. The reaction mixture was stirred at $70^{\circ} \mathrm{C}$ for $3 \mathrm{~h}$. Column chromatography (eluent: 70:29:1, ether:petrol: $\mathrm{Et}_{3} \mathrm{~N}$ to ether) yielded alkenyl $\mathrm{N}$-aminosulfonamide $\mathbf{3 m}$ as an off-white crystalline solid (73 mg, 59\%, Z:E, 20:1); $\mathrm{mp}>300{ }^{\circ} \mathrm{C}\left(\mathrm{CH}_{2} \mathrm{Cl}_{2}\right)$; $v_{\max }($ neat $) / \mathrm{cm}^{-1} 3225,2935,2849,1599,1502,1455,1440,1389$, $1323,1268,1211,1143,1028 ; \delta_{\mathrm{H}}\left(400 \mathrm{MHz}, \mathrm{CDCl}_{3}\right) 7.53(1 \mathrm{H}, \mathrm{s}$, $\operatorname{Ar}(\mathrm{OMe}) H), 7.39-7.32(6 \mathrm{H}, \mathrm{m}, 6 \times \mathrm{Ph} H), 7.31-7.27(4 \mathrm{H}, \mathrm{m}, 4 \times$ $\mathrm{Ph} H), 7.02(1 \mathrm{H}, \mathrm{s}, \operatorname{Ar}(\mathrm{OMe}) H), 6.86(1 \mathrm{H}, \mathrm{d}, J 12.0, \mathrm{ArCH}=\mathrm{CHS})$, $5.80(1 \mathrm{H}, \mathrm{d}, J 12.0, \mathrm{ArCH}=\mathrm{CHS}), 5.34(1 \mathrm{H}, \mathrm{s}, \mathrm{NH}), 3.89(3 \mathrm{H}, \mathrm{s}$, $\mathrm{OMe}$ ) overlapping $3.89(3 \mathrm{H}, \mathrm{s}, \mathrm{OMe}), 3.87\left(4 \mathrm{H}, \mathrm{s}, 2 \times \mathrm{CH}_{2} \mathrm{Ph}\right) ; \delta_{\mathrm{C}}$ $\left(126 \mathrm{MHz}, \mathrm{CDCl}_{3}\right) 151.9(\mathrm{C}), 148.8(\mathrm{C}), 138.1(\mathrm{CH}), 137.9(\mathrm{CH})$, $130.9(4 \times \mathrm{CH}), 129.1(4 \times \mathrm{CH}), 129.0(2 \times \mathrm{C}), 128.5(2 \times \mathrm{CH})$, $126.0(\mathrm{C}), 116.7(\mathrm{CH}), 115.8(\mathrm{CH}), 115.5(\mathrm{C}), 61.6\left(2 \times \mathrm{CH}_{2}\right)$, 56.4(4) $\left.\left(\mathrm{CH}_{3}\right), 56.4(2)\left(\mathrm{CH}_{3}\right) ; \mathrm{m} / z\left(\mathrm{ESI}^{+}\right) 541\left([\mathrm{M}+\mathrm{Na}]^{+}\right), 95 \%\right)$, $539\left([\mathrm{M}+\mathrm{Na}]^{+}, 100 \%\right)$; HRMS $\left(\mathrm{ESI}^{+}\right) \mathrm{C}_{24} \mathrm{H}_{26} \mathrm{O}_{4} \mathrm{~N}_{2} \mathrm{BrS}^{+}([\mathrm{M}+$ $\mathrm{H}]^{+}$) requires 519.0782 and 517.0802 , found 519.0769 and 517.0788 .

\subsubsection{5. (Z)-2-(2-Bromo-4,5-dimethoxyphenyl)- $N$ '-methyl- $N$ - phenylethenesulfonohydrazide (3n)}

Prepared according to the general procedure A using 1-bromo2-(2-iodovinyl)-4,5-dimethoxybenzene ( $89 \mathrm{mg}, 0.24 \mathrm{mmol}, Z: E$, $>20: 1$ ). The reaction mixture was stirred at $70^{\circ} \mathrm{C}$ for $3 \mathrm{~h}$. Column chromatography (eluent: 70:29:1, ether:petrol:Et ${ }_{3} \mathrm{~N}$ to ether) yielded alkenyl $N$-aminosulfonamide $\mathbf{3 n}$ as an off-white solid (78 
$\mathrm{mg}, 76 \%, Z: E, 10: 1) ; \mathrm{mp} 91-94{ }^{\circ} \mathrm{C}\left(\mathrm{CH}_{2} \mathrm{Cl}_{2}\right) ; v_{\max }$ (neat) $/ \mathrm{cm}^{-1} 3226$, 2928, 2852, 1599, 1502, 1464, 1439, 1390, 1337, 1275, 1210, $1182,1152,1029 ; \delta_{\mathrm{H}}\left(400 \mathrm{MHz}, \mathrm{CDCl}_{3}\right) 7.61(1 \mathrm{H}, \mathrm{s}, \mathrm{Ar}(\mathrm{OMe}) H)$, 7.31-7.26 (2H, m, $2 \times \mathrm{Ph} H), 7.21(1 \mathrm{H}, \mathrm{d}, J 12.0, \mathrm{ArC} H=\mathrm{CHS})$, 7.05-7.00 (3H, m, $\operatorname{Ar}(\mathrm{OMe}) H, 2 \times \mathrm{Ph} H), 6.95(1 \mathrm{H}, \mathrm{t}, J 7.5, \mathrm{Ph} H)$, $6.47(1 \mathrm{H}, \mathrm{d}, J 12.0, \mathrm{ArCH}=\mathrm{CHS}), 6.04(1 \mathrm{H}, \mathrm{s}, \mathrm{NH}), 3.88(3 \mathrm{H}, \mathrm{s}$, $\mathrm{OMe}), 3.76(3 \mathrm{H}, \mathrm{s}, \mathrm{OMe}), 3.21(3 \mathrm{H}, \mathrm{s}, \mathrm{NMe}) ; \delta_{\mathrm{C}}(126 \mathrm{MHz}$, $\left.\mathrm{CDCl}_{3}\right) 151.0(\mathrm{C}), 149.8(\mathrm{C}), 147.9(\mathrm{C}), 140.5(\mathrm{CH}), 129.4(2 \times$ $\mathrm{CH}), 126.8(\mathrm{CH}), 124.6(\mathrm{C}), 121.5(\mathrm{CH}), 115.8(\mathrm{C}), 115.0(\mathrm{CH})$, 114.7(3) $(\mathrm{CH}), 114.6(8)(2 \times \mathrm{CH}), 56.3\left(\mathrm{CH}_{3}\right), 56.2\left(\mathrm{CH}_{3}\right), 43.7$ $\left(\mathrm{CH}_{3}\right) ; \mathrm{m} / z\left(\mathrm{ESI}^{+}\right) 451\left([\mathrm{M}+\mathrm{Na}]^{+}, 100 \%\right), 449\left([\mathrm{M}+\mathrm{Na}]^{+}, 95 \%\right)$; HRMS $\left(\mathrm{ESI}^{+}\right) \mathrm{C}_{17} \mathrm{H}_{20} \mathrm{O}_{4} \mathrm{~N}_{2} \mathrm{BrS}^{+}\left([\mathrm{M}+\mathrm{H}]^{+}\right)$requires 429.0312 and 427.0333, found 429.0312 and 427.0332 .

\subsection{Synthesis of benzosultams from alkenylsulfonamides.}

\subsubsection{General procedure B for the preparation of benzosultams 4}

An oven-dried tube was evacuated and backfilled with $\mathrm{N}_{2}$. It was then charged with CataCXium A (20 mol\%), palladium(II) acetate $(10 \mathrm{~mol} \%)$, and $\mathrm{Cs}_{2} \mathrm{CO}_{3}$ (1.5 equiv.). The solid reagents were weighed out in the air. The tube was then evacuated and backfilled with $\mathrm{N}_{2}$. 1,4-ioxane $[0.05 \mathrm{M}]$ was added via syringe through a suba-seal under $\mathrm{N}_{2}$. The reaction mixture was stirred for 10 minutes. A solution of the alkenyl $N$-aminosulfonamide (1 equiv.) in 1,4-dioxane [0.1 M] was added via microsyringe. The reaction mixture was stirred at $100^{\circ} \mathrm{C}$ for $1 \mathrm{~h}$. After cooling to $\mathrm{RT}$, the suspension was filtered through a short pad of Celite and the residue washed sequentially with $\mathrm{CH}_{2} \mathrm{Cl}_{2}\left(20 \mathrm{~mL} \mathrm{mmol}^{-1}\right)$ and ether $\left(20 \mathrm{~mL} \mathrm{mmol}^{-1}\right)$ before being concentrated in vacuo. Purification via column chromatography yielded the corresponding alkenyl $\mathrm{N}$-aminobenzosultam.

\subsubsection{1-(Morpholin-4-yl)-1H-2,1-benzothiazine 2,2-dioxide (4a)}

Prepared according to the general procedure B using $(Z)-2-(2-$ bromophenyl)- $N$-(morpholin-4-yl)ethenesulfonamide 3a (50 mg, $0.14 \mathrm{mmol}, Z: E, 12: 1)$. Column chromatography (eluent: 3:7, petrol:ether) yielded benzothiazine 2,2-dioxide $\mathbf{4 a}$ as a white crystalline solid (32 mg, 83\%); mp 150-153 ${ }^{\circ} \mathrm{C}\left(\mathrm{CH}_{2} \mathrm{Cl}_{2}\right) ; v_{\max }$ (neat) $/ \mathrm{cm}^{-1} 2955,2915,2863,1612,1559,1453,1360,1320,1270$, $1213,1159,1145,1111,1070,1037 ; \delta_{\mathrm{H}}\left(500 \mathrm{MHz},\left(\mathrm{CD}_{3}\right)_{2} \mathrm{SO}\right.$, $363 \mathrm{~K}) 7.74(1 \mathrm{H}, \mathrm{d}, J 8.0, \mathrm{ArH}), 7.61-7.53(2 \mathrm{H}, \mathrm{m}, 2 \times \mathrm{Ar} H), 7.50$ $(1 \mathrm{H}, \mathrm{d}, J$ 10.0, $\mathrm{ArCH}=\mathrm{CHS}), 7.25-7.19(1 \mathrm{H}, \mathrm{m}, \mathrm{Ar} H)$ overlapping $7.19(1 \mathrm{H}, \mathrm{d}, J$ 10.0, $\mathrm{ArCH}=\mathrm{CHS}), 3.74(4 \mathrm{H}$, br. t, $J 4.5$, $\left.\mathrm{N}\left(\mathrm{CH}_{2} \mathrm{CH}_{2}\right)_{2} \mathrm{O}\right), 3.43\left(4 \mathrm{H}\right.$, br. s, $\left.\mathrm{N}\left(\mathrm{CH}_{2} \mathrm{CH}_{2}\right)_{2} \mathrm{O}\right) ; \delta_{\mathrm{C}}(126 \mathrm{MHz}$, $\left.\mathrm{CDCl}_{3}\right) 141.5(\mathrm{C}), 135.6(\mathrm{CH}), 131.5(\mathrm{CH}), 129.9(\mathrm{CH}), 125.8$ $(\mathrm{CH}), 123.0(\mathrm{CH}), 120.5(\mathrm{C}), 116.9(\mathrm{CH}), 67.1\left(2 \times \mathrm{CH}_{2}\right), 53.5(2$ $\left.\times \mathrm{CH}_{2}\right) ; \mathrm{m} / z\left(\mathrm{ESI}^{+}\right) 289\left([\mathrm{M}+\mathrm{Na}]^{+}, 100 \%\right), 267\left([\mathrm{M}+\mathrm{H}]^{+}, 45 \%\right)$; HRMS $\left(\mathrm{ESI}^{+}\right) \mathrm{C}_{12} \mathrm{H}_{14} \mathrm{~N}_{2} \mathrm{NaO}_{3} \mathrm{~S}^{+}\left([\mathrm{M}+\mathrm{Na}]^{+}\right)$requires 289.0617, found 289.0617 .

\subsubsection{1-(Morpholin-4-yl)-6-(trifluoromethyl)-1H-2,1- benzothiazine 2,2-dioxide (4b)}

Prepared according to the general procedure B using 2-(2bromo-5-(trifluoromethyl)phenyl)- $N$ -

morpholinoethenesulfonamide $\mathbf{3 b}$ (68 mg, $0.16 \mathrm{mmol}, Z: E, 15: 1)$. Column chromatography (eluent: $3: 7$, petrol:ether) yielded benzothiazine 2,2-dioxide $\mathbf{4 b}$ as a white crystalline solid (41 $\mathrm{mg}$, $75 \%) ; \mathrm{mp} 120-122{ }^{\circ} \mathrm{C}\left(\mathrm{CH}_{2} \mathrm{Cl}_{2}\right) ; v_{\max }$ (neat) $/ \mathrm{cm}^{-1} 3082,2869$, $1620,1359,1320,1299,1277,1202,1162,1133,1104,1075$, 1029,$1012 ; \delta_{\mathrm{H}}\left(500 \mathrm{MHz},\left(\mathrm{CD}_{3}\right)_{2} \mathrm{SO}, 363 \mathrm{~K}\right) 8.06(1 \mathrm{H}, \mathrm{s}, \mathrm{ArH})$, $7.96(1 \mathrm{H}, \mathrm{d}, J 9.0, \operatorname{Ar} H), 7.86(1 \mathrm{H}, \mathrm{d}, J 9.0, \operatorname{Ar} H), 7.68(1 \mathrm{H}, \mathrm{d}$, $J$ 10.5, $\mathrm{ArCH}=\mathrm{CHS}), 7.41(1 \mathrm{H}, \mathrm{d}, J 10.5, \mathrm{ArCH}=\mathrm{CHS}), 3.78(4 \mathrm{H}$, br. s, $\left.\mathrm{N}\left(\mathrm{CH}_{2} \mathrm{CH}_{2}\right)_{2} \mathrm{O}\right), 3.47\left(4 \mathrm{H}\right.$, br. s, $\left.\mathrm{N}\left(\mathrm{CH}_{2} \mathrm{CH}_{2}\right)_{2} \mathrm{O}\right) ; \delta_{\mathrm{C}}(126$
$\left.\mathrm{MHz}, \mathrm{CDCl}_{3}\right) 144.5(\mathrm{C}), 134.6(\mathrm{CH}), 128.1\left(\mathrm{CH}, \mathrm{q}, J_{\mathrm{CF}} 3.5\right)$, $127.1\left(\mathrm{CH}, \mathrm{q}, J_{\mathrm{CF}} 3.5\right), 126.0(\mathrm{CH}), 125.2\left(\mathrm{C}, \mathrm{q}, J_{\mathrm{CF}} 33.0\right), 123.6$ $\left(\mathrm{C}, \mathrm{q}, J_{\mathrm{CF}} 272.0\right), 119.7(\mathrm{C}), 117.1(\mathrm{CH}), 67.8\left(2 \times \mathrm{CH}_{2}\right), 53.9(2 \times$ $\left.\mathrm{CH}_{2}\right) ; \delta_{\mathrm{F}}\left(377 \mathrm{MHz}, \mathrm{CDCl}_{3}\right)-62.1\left(\mathrm{~s}, \mathrm{ArCF}_{3}\right)\left\{{ }^{1} \mathrm{H}\right\} ; m / z\left(\mathrm{ESI}^{+}\right) 357$ $\left([\mathrm{M}+\mathrm{Na}]^{+}, 100 \%\right) ; 334\left([\mathrm{M}+\mathrm{H}]^{+}, 30 \%\right) ; \operatorname{HRMS}\left(\mathrm{ESI}^{+}\right)$ $\mathrm{C}_{13} \mathrm{H}_{13} \mathrm{~F}_{3} \mathrm{~N}_{2} \mathrm{NaO}_{3} \mathrm{~S}^{+}\left([\mathrm{M}+\mathrm{Na}]^{+}\right)$requires 357.0491, found 357.0477 .

\subsubsection{7-Fluoro-1-morpholino-1H-benzo[c][1,2]thiazine 2,2- dioxide $(4 \mathrm{c})$}

Prepared according to the general procedure B using 2-(2bromo-4-fluorophenyl)- $N$-morpholinoethenesulfonamide $3 \mathbf{c}$ (50 mg, 0.14 mmol, Z:E, 20:1). Column chromatography (eluent: 3:7, petrol:ether) yielded benzothiazine 2,2-dioxide $\mathbf{4 c}$ as a white crystalline solid $(27 \mathrm{mg}, 69 \%) ; \mathrm{mp}>250{ }^{\circ} \mathrm{C}\left(\mathrm{CH}_{2} \mathrm{Cl}_{2}\right) ; v_{\max }$ (neat) $/ \mathrm{cm}^{-1} 2918,2853,1617,1567,1493,1457,1315,1268,1214$, 1164,$1107 ; \delta_{\mathrm{H}}\left(500 \mathrm{MHz},\left(\mathrm{CD}_{3}\right)_{2} \mathrm{SO}, 363 \mathrm{~K}\right) 7.67(1 \mathrm{H}, \mathrm{dd}, J 8.5$, 6.5, $\mathrm{ArH}), 7.52(1 \mathrm{H}, \mathrm{d}, J 10.0, \mathrm{ArCH}=\mathrm{CHS})$ overlapping 7.52-7.49 $(1 \mathrm{H}, \mathrm{m}, \mathrm{ArH}), 7.18(1 \mathrm{H}, \mathrm{d}, J 10.0, \mathrm{ArCH}=\mathrm{CHS}), 7.04(1 \mathrm{H}, \mathrm{td}, J$ 8.5, 3.0, $\mathrm{ArH}), 3.76\left(3 \mathrm{H}\right.$, br. t, $\left.J 4.5, \mathrm{~N}\left(\mathrm{CH}_{2} \mathrm{CH}_{2}\right)_{2} \mathrm{O}\right), 3.45(3 \mathrm{H}$, br. $\left.\mathrm{s}, \mathrm{N}\left(\mathrm{CH}_{2} \mathrm{CH}_{2}\right)_{2} \mathrm{O}\right) ; \delta_{\mathrm{C}}\left(126 \mathrm{MHz},\left(\mathrm{CD}_{3}\right)_{2} \mathrm{SO}\right) 163.4\left(\mathrm{~d}, J_{\mathrm{CF}} 248.0\right)$, $143.4\left(\mathrm{~d}, J_{\mathrm{CF}} 12.0\right), 134.4,132.1$ (d, $\left.J_{\mathrm{CF}} 10.0\right), 123.9\left(\mathrm{~d}, J_{\mathrm{CF}} 3.0\right)$, $116.6\left(\mathrm{~d}, J_{\mathrm{CF}} 3.0\right), 110.0\left(\mathrm{~d}, J_{\mathrm{CF}} 23.0\right), 103.1\left(\mathrm{~d}, J_{\mathrm{CF}} 28.0\right), 66.6(2 \mathrm{C})$, $53.1(2 \mathrm{C}) ; \delta_{\mathrm{F}}\left(377 \mathrm{MHz}, \mathrm{CDCl}_{3}\right)-105.8(\mathrm{~s}, \mathrm{Ar} F)\left\{{ }^{1} \mathrm{H}\right\}$; HRMS $\left(\mathrm{FI}^{+}\right) \mathrm{C}_{12} \mathrm{H}_{13} \mathrm{FN}_{2} \mathrm{O}_{3} \mathrm{~S}^{+}\left([\mathrm{M}]^{+}\right)$requires 284.0631 and 284.0626.

\subsubsection{6-Fluoro-1-morpholino-1H-benzo[c][1,2]thiazine 2,2- dioxide (4d)}

Prepared according to the general procedure B using 2-(2bromo-5-fluorophenyl)- $N$-morpholinoethenesulfonamide 3d (42 $\mathrm{mg}, 0.11 \mathrm{mmol}, Z: E, 15: 1$ ). Column chromatography (eluent: 3:7, petrol:ether) yielded benzothiazine 2,2-dioxide $4 \mathbf{d}$ as a white crystalline solid (25 mg, 78\%); mp $145-148{ }^{\circ} \mathrm{C} ; v_{\max }$ (neat) $/ \mathrm{cm}^{-1}$ 3078, 2959, 2923, 2850, 1608, 1564, 1471, 1426, 1370, 1346, $1318,1265,1239,1157,1109,1071,1029,1012 ; \delta_{\mathrm{H}}(500 \mathrm{MHz}$, $\left.\left(\mathrm{CD}_{3}\right)_{2} \mathrm{SO}, 363 \mathrm{~K}\right) 7.76(1 \mathrm{H}, \mathrm{dd}, J 9.0,5.0, \mathrm{Ar} H), 7.50(1 \mathrm{H}, \mathrm{d}, J$ 10.0, $\mathrm{ArCH}=\mathrm{CHS})$ overlapping $7.48(1 \mathrm{H}, \mathrm{dd}, J 9.0,3.0, \mathrm{Ar} H)$, 7.43-7.37 (1H, m, ArH), $7.30(1 \mathrm{H}, \mathrm{d}, J 10.0, \mathrm{ArCH}=\mathrm{CHS}), 3.73$ $\left(4 \mathrm{H}, \quad \mathrm{t}, \quad J 4.5, \quad \mathrm{~N}\left(\mathrm{CH}_{2} \mathrm{CH}_{2}\right)_{2} \mathrm{O}\right), 3.42(4 \mathrm{H}, \quad$ br. $\mathrm{t}, J 4.5$, $\left.\mathrm{N}\left(\mathrm{CH}_{2} \mathrm{CH}_{2}\right)_{2} \mathrm{O}\right) ; \delta_{\mathrm{C}}\left(126 \mathrm{MHz},\left(\mathrm{CD}_{3}\right)_{2} \mathrm{SO}\right) 157.6\left(\mathrm{C}, \mathrm{d}, J_{\mathrm{CF}} 241.5\right)$, $138.0(\mathrm{C}), 134.5\left(\mathrm{CH}, \mathrm{d}, J_{\mathrm{CF}} 2.0\right), 127.5(\mathrm{CH}), 121.9\left(\mathrm{C}, \mathrm{d}, J_{\mathrm{CF}} 9.0\right)$, $120.0\left(\mathrm{CH}, \mathrm{d}, J_{\mathrm{CF}} 8.0\right), 118.6\left(\mathrm{CH}, \mathrm{d}, J_{\mathrm{CF}} 23.0\right), 115.1\left(\mathrm{CH}, \mathrm{d}, J_{\mathrm{CF}}\right.$ 23.7), $67.1\left(2 \times \mathrm{CH}_{2}\right), 53.4\left(2 \times \mathrm{CH}_{2}\right) ; \delta_{\mathrm{F}}\left(377 \mathrm{MHz},\left(\mathrm{CD}_{3}\right)_{2} \mathrm{SO}\right)-$ $120.2(\mathrm{~s}, \mathrm{Ar} F)\left\{{ }^{1} \mathrm{H}\right\}$; HRMS $\left(\mathrm{FI}^{+}\right) \mathrm{C}_{12} \mathrm{H}_{13} \mathrm{FN}_{2} \mathrm{O}_{3} \mathrm{~S}^{+}\left([\mathrm{M}]^{+}\right.$requires 284.0631 and 284.0630 .

\subsubsection{1-Morpholino-1H-naphtho[1,2-c][1,2] thiazine 2,2-dioxide (4e)}

Prepared according to the general procedure B using 2-(1bromonaphthalen-2-yl)- $N$-morpholinoethenesulfonamide 3 e (60 $\mathrm{mg}, 0.15 \mathrm{mmol}, Z: E, 15: 1)$. Column chromatography (eluent: 3:7, petrol:ether) yielded benzothiazine 2,2-dioxide $4 \mathbf{e}$ as a white crystalline solid (32 mg, 68\%); mp 204-207 ${ }^{\circ} \mathrm{C}\left(\mathrm{CH}_{2} \mathrm{Cl}_{2}\right) ; v_{\max }$ (neat) $/ \mathrm{cm}^{-1} 3058,2916,2851,1593,1504,1459,1371,1335,1305$, $1261,1205,1162,1130,1102,1019 ; \delta_{\mathrm{H}}\left(500 \mathrm{MHz},\left(\mathrm{CD}_{3}\right)_{2} \mathrm{SO}, 363\right.$ K) $8.24(1 \mathrm{H}, \mathrm{d}, J 8.0, \mathrm{Ar} H), 7.98(1 \mathrm{H}, \mathrm{d}, J 8.0, \operatorname{Ar} H), 7.91(1 \mathrm{H}, \mathrm{d}$, $J$ 8.5, $\mathrm{ArH}), 7.70-7.59(4 \mathrm{H}, \mathrm{m}, \mathrm{ArCH}=\mathrm{CHS}, 3 \times \mathrm{ArH}) 7.25(1 \mathrm{H}, \mathrm{d}$, $J$ 10.0, $\mathrm{ArCH}=\mathrm{CHS}), 3.45\left(4 \mathrm{H}\right.$, br. t, $\left.J 4.5, \mathrm{~N}\left(\mathrm{CH}_{2} \mathrm{CH}_{2}\right)_{2} \mathrm{O}\right), 3.36$ (4H, br. t, $\left.J 4.5, \mathrm{~N}\left(\mathrm{CH}_{2} \mathrm{CH}_{2}\right)_{2} \mathrm{O}\right) ; \delta_{\mathrm{C}}\left(126 \mathrm{MHz},\left(\mathrm{CD}_{3}\right)_{2} \mathrm{SO}\right) 137.0$ $(\mathrm{CH}), 136.9(\mathrm{C}), 134.4(\mathrm{C}), 128.8(\mathrm{C}), 128.4(\mathrm{CH}), 128.2(\mathrm{CH})$, $127.8(\mathrm{CH}), 127.4(\mathrm{CH}), 127.3(\mathrm{CH}), 125.3(\mathrm{CH}), 124.6(\mathrm{CH})$, 
$122.8(\mathrm{C}), 67.0\left(2 \times \mathrm{CH}_{2}\right), 52.2\left(2 \times \mathrm{CH}_{2}\right) ; \mathrm{HRMS}\left(\mathrm{FI}^{+}\right)$ $\mathrm{C}_{16} \mathrm{H}_{16} \mathrm{~N}_{2} \mathrm{O}_{3} \mathrm{~S}^{+}\left([\mathrm{M}]^{+}\right)$requires 316.0882 , found 316.0894 .

\subsubsection{1-Morpholino-1H-[1,3]dioxolo[4',5':4,5]benzo[1,2- c][1,2] thiazine 2,2-dioxide (4f)}

Prepared according to the general procedure B using (Z)-2-(6bromobenzo[d][1,3]dioxol-5-yl)- $N$ -

morpholinoethenesulfonamide 3f (62 mg, $0.16 \mathrm{mmol}, Z: E, 20: 1)$. Column chromatography (eluent: $3: 7$, petrol:ether) yielded benzothiazine 2,2-dioxide $\mathbf{4 f}$ as a white crystalline solid (39 $\mathrm{mg}$, $80 \%)$; mp 188-189 ${ }^{\circ} \mathrm{C}\left(\mathrm{CH}_{2} \mathrm{Cl}_{2}\right) ; v_{\max }$ (neat) $/ \mathrm{cm}^{-1} 2912,2852$, $1625,1574,1499,1475,1432,1383,1311,1260,1241,1221$, $1180,1156,1124,1103,1073,1037 ; \delta_{\mathrm{H}}\left(500 \mathrm{MHz},\left(\mathrm{CD}_{3}\right)_{2} \mathrm{SO}, 363\right.$ K) $7.36(1 \mathrm{H}, \mathrm{d}, J 10.0, \mathrm{ArCH}=\mathrm{CHS}), 7.28(1 \mathrm{H}, \mathrm{s}, \mathrm{Ar} H), 7.10$ $(1 \mathrm{H}, \mathrm{s}, \mathrm{ArH}), 7.00(1 \mathrm{H}, \mathrm{d}, J 10.0, \mathrm{ArCH}=\mathrm{CHS}), 6.10(2 \mathrm{H}, \mathrm{s}$, $\left.\mathrm{OCH}_{2} \mathrm{O}\right), 3.72\left(4 \mathrm{H}\right.$, br. t, $\left.J 4.5, \mathrm{~N}\left(\mathrm{CH}_{2} \mathrm{CH}_{2}\right)_{2} \mathrm{O}\right), 3.39(4 \mathrm{H}$, br. s, $\left.\mathrm{N}\left(\mathrm{CH}_{2} \mathrm{CH}_{2}\right)_{2} \mathrm{O}\right) ; \delta_{\mathrm{C}}\left(126 \mathrm{MHz},\left(\mathrm{CD}_{3}\right)_{2} \mathrm{SO}\right) 150.2(\mathrm{C}), 143.7(\mathrm{C})$, $138.1(\mathrm{C}), 135.3(\mathrm{CH}), 122.8(\mathrm{CH}), 114.6(\mathrm{C}), 107.6(\mathrm{CH}), 102.0$ $\left(\mathrm{CH}_{2}\right), 99.0(\mathrm{CH}), 67.0\left(2 \times \mathrm{CH}_{2}\right), 53.3\left(2 \times \mathrm{CH}_{2}\right) ; \mathrm{HRMS}\left(\mathrm{FI}^{+}\right)$ $\mathrm{C}_{13} \mathrm{H}_{14} \mathrm{~N}_{2} \mathrm{O}_{5} \mathrm{~S}^{+}\left([\mathrm{M}]^{+}\right)$requires 310.0623 , found 310.0625 .

\subsubsection{6-(Benzyloxy)-1-morpholino-1H-benzo[c][1,2]thiazine 2,2-dioxide (4g)}

Prepared according to the general procedure B using 2-(5(benzyloxy)-2-bromophenyl)- $N$-morpholinoethenesulfonamide $3 g$ (75 mg, $0.17 \mathrm{mmol}, Z: E, 8: 1)$. Column chromatography (eluent: 3:7, petrol:ether) yielded benzothiazine 2,2-dioxide $\mathbf{4 g}$ as a white crystalline solid (49 mg, 79\%); mp 195-198 ${ }^{\circ} \mathrm{C}$ (dec.); $v_{\max }$ (neat) $/ \mathrm{cm}^{-1} 2923,2852,1599,1564,1455,1332,1270,1243,1161$, 1111,$1013 ; \delta_{\mathrm{H}}\left(500 \mathrm{MHz}, \mathrm{CDCl}_{3}\right) 7.61(1 \mathrm{H}, \mathrm{d}, J 9.0, \mathrm{ArH}), 7.44-$ $7.34(5 \mathrm{H}, \mathrm{m}, 5 \times \mathrm{OBn} H), 7.14(1 \mathrm{H}, \mathrm{d}, J 10.0, \mathrm{ArCH}=\mathrm{CHS})$ overlapping 7.16-7.12 (1H, m, ArH), $6.94(1 \mathrm{H}, \mathrm{d}, J 2.5, \mathrm{Ar} H), 6.78$ $(1 \mathrm{H}, \mathrm{d}, J 10.0, \mathrm{ArCH}=\mathrm{CHS}), 5.08\left(2 \mathrm{H}, \mathrm{s}, \mathrm{OCH}_{2} \mathrm{Ph}\right), 3.80(4 \mathrm{H}$, br. s, $\left.\mathrm{N}\left(\mathrm{CH}_{2} \mathrm{CH}_{2}\right)_{2} \mathrm{O}\right), 3.51\left(4 \mathrm{H}\right.$, br. s, $\left.\mathrm{N}\left(\mathrm{CH}_{2} \mathrm{CH}_{2}\right)_{2} \mathrm{O}\right) ; \delta_{\mathrm{C}}(126 \mathrm{MHz}$, $\left.\mathrm{CDCl}_{3}\right) 154.8(\mathrm{C}), 136.5(\mathrm{C}), 135.9(\mathrm{C}), 135.2(\mathrm{CH}), 128.8(2 \times$ $\mathrm{CH}), 128.3(\mathrm{CH}), 127.5(2 \times \mathrm{CH}), 126.0(\mathrm{CH}), 121.8(\mathrm{C}), 119.9$ $(\mathrm{CH}), 119.6(\mathrm{CH}), 113.8(\mathrm{CH}), 70.7\left(\mathrm{CH}_{2}\right), 68.0\left(2 \times \mathrm{CH}_{2}\right), 53.8$ $\left(2 \times \mathrm{CH}_{2}\right)$; HMRS $\left(\mathrm{FI}^{+}\right) \mathrm{C}_{19} \mathrm{H}_{20} \mathrm{~N}_{2} \mathrm{O}_{4} \mathrm{~S}^{+}\left([\mathrm{M}]^{+}\right)$requires 372.1144, found 372.1140 .

\subsubsection{7-Methyl-1-morpholino-1H-benzo[c][1,2]thiazine 2,2- dioxide (4h)}

Prepared according to the general procedure B using 2-(2bromo-4-methylphenyl)- $N$-morpholinoethenesulfonamide $\mathbf{3 h}$ (53 $\mathrm{mg}, 0.15 \mathrm{mmol}, Z: E, 15: 1$ ). Column chromatography (eluent: 3:7, petrol:ether) yielded benzothiazine 2,2-dioxide $\mathbf{4 h}$ as a white crystalline solid (29 mg, 71\%); $\mathrm{mp} 115-118{ }^{\circ} \mathrm{C}\left(\mathrm{CH}_{2} \mathrm{Cl}_{2}\right) ; v_{\max }$ (neat) $/ \mathrm{cm}^{-1}$ 2957, 2920, 2854, 1613, 1550, 1488, 1452, 1362, 1319, $1268,1213,1159,1146,1110,1071,1039 ; \delta_{\mathrm{H}}(500 \mathrm{MHz}$, $\left.\left(\mathrm{CD}_{3}\right)_{2} \mathrm{SO}, 363 \mathrm{~K}\right) 7.53(1 \mathrm{H}, \mathrm{s}, \mathrm{ArH}), 7.49-7.42(2 \mathrm{H}, \mathrm{m}, \mathrm{ArH}$, $\mathrm{ArCH}=\mathrm{CHS}), 7.09(1 \mathrm{H}, \mathrm{d}, J 10.0, \mathrm{ArCH}=\mathrm{CHS}), 7.04(1 \mathrm{H}, \mathrm{d}, J 7.5$, $\mathrm{ArH}), 3.74\left(4 \mathrm{H}\right.$, br. t, $\left.J 4.5, \mathrm{~N}\left(\mathrm{CH}_{2} \mathrm{CH}_{2}\right)_{2} \mathrm{O}\right), 3.42(4 \mathrm{H}$, br. s, $\left.\mathrm{N}\left(\mathrm{CH}_{2} \mathrm{CH}_{2}\right)_{2} \mathrm{O}\right), 2.43(3 \mathrm{H}, \mathrm{s}, \mathrm{ArMe}) ; \delta_{\mathrm{C}}\left(126 \mathrm{MHz},\left(\mathrm{CD}_{3}\right)_{2} \mathrm{SO}\right)$ $141.9(\mathrm{C}), 141.5(\mathrm{C}), 135.5(\mathrm{CH}), 129.9(\mathrm{CH}), 124.7(\mathrm{CH}), 124.1$ $(\mathrm{CH}), 118.1(\mathrm{C}), 117.0(\mathrm{CH}), 67.0\left(2 \times \mathrm{CH}_{2}\right), 53.4\left(2 \times \mathrm{CH}_{2}\right), 21.6$ $\left(\mathrm{CH}_{3}\right)$; HRMS $\left(\mathrm{FI}^{+}\right) \mathrm{C}_{13} \mathrm{H}_{16} \mathrm{~N}_{2} \mathrm{O}_{3} \mathrm{~S}^{+}\left([\mathrm{M}]^{+}\right)$requires 280.0882, found 280.0887 .
Prepared according to the general procedure B using 2-(2bromo-4,5-dimethoxyphenyl)- $N$-morpholinoethenesulfonamide 3i (70 mg, $0.17 \mathrm{mmol}, Z: E, 15: 1)$. Column chromatography (eluent: 3:7, petrol:ether) yielded benzothiazine 2,2-dioxide $\mathbf{4 i}$ as a white crystalline solid (48 $\mathrm{mg}, 85 \%)$; $\mathrm{mp} \quad 189-190{ }^{\circ} \mathrm{C}$ $\left(\mathrm{CH}_{2} \mathrm{Cl}_{2}\right.$ :petrol, 1:1); $v_{\max }$ (neat) $/ \mathrm{cm}^{-1} 3046,2851,1613,1557$, $1501,1459,1363,1302,1276,1239,1203,1181,1152,1133$, $1112,1039,1004 ; \delta_{\mathrm{H}}\left(500 \mathrm{MHz},\left(\mathrm{CD}_{3}\right)_{2} \mathrm{SO}, 363 \mathrm{~K}\right) 7.37(1 \mathrm{H}, \mathrm{d}, J$ 10.0, $\mathrm{ArCH}=\mathrm{CHS}), 7.26(1 \mathrm{H}, \mathrm{s}, \mathrm{Ar} H), 7.15(1 \mathrm{H}, \mathrm{s}, \operatorname{Ar} H), 6.96(1 \mathrm{H}$, $\mathrm{d}, J 10.0, \mathrm{ArCH}=\mathrm{CHS}), 3.90(3 \mathrm{H}, \mathrm{s}, \mathrm{OMe}), 3.80(3 \mathrm{H}, \mathrm{s}, \mathrm{OMe}), 3.72$ $\left(4 \mathrm{H}\right.$, br. t, $\left.J 4.5, \mathrm{~N}\left(\mathrm{CH}_{2} \mathrm{CH}_{2}\right)_{2} \mathrm{O}\right), 3.39(4 \mathrm{H}$, br. t, $J 4.5$, $\left.\mathrm{N}\left(\mathrm{CH}_{2} \mathrm{CH}_{2}\right)_{2} \mathrm{O}\right) ; \delta_{\mathrm{C}}\left(126 \mathrm{MHz}, \mathrm{CDCl}_{3}\right) 152.3(\mathrm{C}), 145.8(\mathrm{C}), 137.3$ (C), $135.2(\mathrm{CH}), 122.3(\mathrm{CH}), 113.7(\mathrm{C}), 111.1(\mathrm{CH}), 101.2(\mathrm{CH})$, $68.2\left(2 \times \mathrm{CH}_{2}\right), 56.5\left(\mathrm{CH}_{3}\right), 56.2\left(\mathrm{CH}_{3}\right), 53.9\left(2 \times \mathrm{CH}_{2}\right)$; HMRS $\left(\mathrm{FI}^{+}\right) \mathrm{C}_{14} \mathrm{H}_{18} \mathrm{~N}_{2} \mathrm{O}_{5} \mathrm{~S}^{+}\left([\mathrm{M}]^{+}\right)$requires 326.0928, found 326.0937.

\subsubsection{1-Morpholino-1H-pyrido[2,3-c][1,2]thiazine 2,2-dioxide (4k)}

Prepared according to the general procedure B using 2-(2bromopyridin-3-yl)- $N$-morpholinoethenesulfonamide 3k $(54 \mathrm{mg}$, $0.16 \mathrm{mmol}, Z: E,>20: 1$ ). Column chromatography (eluent: 3:7, petrol:ether) yielded benzothiazine 2,2-dioxide $\mathbf{4 k}$ as a yellow crystalline solid (32 mg, 77\%); mp 240-243 ${ }^{\circ} \mathrm{C}\left(\mathrm{CH}_{2} \mathrm{Cl}_{2}\right) ; v_{\max }$ (neat) $/ \mathrm{cm}^{-1} 3054,2923,2866,1622,1588,1560,1458,1420,1363$, $1304,1271,1250,1171,1154,1127,1104,1013 ; \delta_{\mathrm{H}}(500 \mathrm{MHz}$, $\left.\left(\mathrm{CD}_{3}\right)_{2} \mathrm{SO}, 363 \mathrm{~K}\right) 8.49(1 \mathrm{H}, \mathrm{dd}, J 5.0,2.0, \mathrm{Ar} H), 8.03(1 \mathrm{H}, \mathrm{dd}, J$ 7.5, 2.0, ArH), $7.50(1 \mathrm{H}, \mathrm{d}, J 10.5, \mathrm{ArCH}=\mathrm{CHS}), 7.25(1 \mathrm{H}, \mathrm{d}, J$ 10.5, $\mathrm{ArCH}=\mathrm{CHS})$ overlapping $7.23(1 \mathrm{H}, \mathrm{dd}, J 7.5,5.0, \mathrm{Ar} H)$, 3.79-3.73 (4H, m, N( $\left.\left.\mathrm{CH}_{2} \mathrm{CH}_{2}\right)_{2} \mathrm{O}\right), 3.57\left(4 \mathrm{H}\right.$, br. s, $\left.\mathrm{N}\left(\mathrm{CH}_{2} \mathrm{CH}_{2}\right)_{2} \mathrm{O}\right)$; $\delta_{\mathrm{C}}\left(126 \mathrm{MHz},\left(\mathrm{CD}_{3}\right)_{2} \mathrm{SO}\right) 151.9(\mathrm{C}), 149.2(\mathrm{CH}), 139.4(\mathrm{CH})$, $131.8(\mathrm{CH}), 122.5(\mathrm{CH}), 118.3(\mathrm{CH}), 113.9(\mathrm{C}), 66.8\left(2 \times \mathrm{CH}_{2}\right)$, $52.8\left(2 \times \mathrm{CH}_{2}\right)$; HRMS $\left(\mathrm{FI}^{+}\right) \mathrm{C}_{11} \mathrm{H}_{13} \mathrm{~N}_{3} \mathrm{O}_{3} \mathrm{~S}^{+}\left([\mathrm{M}]^{+}\right)$requires 267.0677, found 267.0681 .

\subsubsection{6,7-Dimethoxy-1-(4-methylpiperazin-1-yl)-1H- benzo[c][1,2]thiazine 2,2-dioxide (4l)}

Prepared according to the general procedure B using 2-(2bromo-4,5-dimethoxyphenyl)- $N$-(4-methylpiperazin-1-

yl)ethenesulfonamide 31 (60 mg, $0.14 \mathrm{mmol}, Z: E, 11: 1)$. Column chromatography (eluent: $1 \% \mathrm{MeOH}$ in $\mathrm{CH}_{2} \mathrm{Cl}_{2}$ ) yielded benzothiazine 2,2-dioxide $\mathbf{4 l}$ as a yellow solid (40 $\mathrm{mg}, 82 \%$ ); $\mathrm{mp}$ 218-220 ${ }^{\circ} \mathrm{C}\left(\mathrm{CH}_{2} \mathrm{Cl}_{2}\right) ; v_{\max }$ (neat) $/ \mathrm{cm}^{-1} 2940,2739,2603,2495$, $1510,1475,1398,1245,1206,1171,1073,1036 ; \delta_{\mathrm{H}}(200 \mathrm{MHz}$, $\left.\mathrm{CD}_{3} \mathrm{CN}\right) 7.26(1 \mathrm{H}, \mathrm{s}, \mathrm{Ar} H)$ overlapping $7.25(1 \mathrm{H}, \mathrm{d}, J 10.0$, $\mathrm{ArCH}=\mathrm{CHS}), 7.00(1 \mathrm{H}, \mathrm{s}, \mathrm{ArH}), 6.71(1 \mathrm{H}, \mathrm{d}, J 10.0, \mathrm{ArCH}=\mathrm{CHS})$, $3.90(3 \mathrm{H}, \mathrm{s}, \mathrm{OMe}), 3.81(3 \mathrm{H}, \mathrm{s}, \mathrm{OMe}), 3.42(4 \mathrm{H}$, br. s, $\left.\mathrm{N}\left(\mathrm{CH}_{2} \mathrm{CH}_{2}\right)_{2} \mathrm{NMe}\right), 2.52\left(4 \mathrm{H}\right.$, br. s, $\left.\mathrm{N}\left(\mathrm{CH}_{2} \mathrm{CH}_{2}\right)_{2} \mathrm{NMe}\right), 2.25(3 \mathrm{H}$, s, NMe); $\delta_{\mathrm{C}}\left(126 \mathrm{MHz}, \mathrm{CD}_{3} \mathrm{CN}\right) 153.2(\mathrm{C}), 146.5(\mathrm{C}), 138.1(\mathrm{C})$, $136.3(\mathrm{CH}), 123.2(\mathrm{CH}), 114.5(\mathrm{C}), 112.3(\mathrm{CH}), 102.3(\mathrm{CH}), 56.7$ $\left(\mathrm{CH}_{3}\right), 53.6\left(\mathrm{CH}_{3}\right), 46.0\left(\mathrm{CH}_{3}\right), 37.3\left(\mathrm{CH}_{2}\right), 37.2\left(\mathrm{CH}_{2}\right), 28.8\left(\mathrm{CH}_{2}\right)$, $28.7\left(\mathrm{CH}_{2}\right) ; m / z\left(\mathrm{ESI}^{+}\right) 340\left([\mathrm{M}+\mathrm{H}]^{+}, 100 \%\right) ; \mathrm{HMRS}\left(\mathrm{ESI}^{+}\right)$ $\mathrm{C}_{15} \mathrm{H}_{22} \mathrm{~N}_{3} \mathrm{O}_{4} \mathrm{~S}^{+}\left([\mathrm{M}+\mathrm{H}]^{+}\right)$requires 340.1326 , found 340.1321 .

\subsubsection{1-(Dibenzylamino)-6,7-dimethoxy-1H- benzo[c][1,2] thiazine 2,2-dioxide (4m)}

Prepared according to the general procedure B using $N^{\prime}, N^{\prime}$ dibenzyl-2-(2-bromo-4,5-

dimethoxyphenyl)ethenesulfonohydrazide 3m (55 mg, $0.11 \mathrm{mmol}$, $Z: E, 20: 1)$. Column chromatography (eluent: $3: 7$, petrol:ether) yielded benzothiazine 2,2-dioxide $\mathbf{4 m}$ as a pale yellow crystalline solid (35 mg, 75\%); mp $190{ }^{\circ} \mathrm{C}$ (dec.) $\left(\mathrm{CH}_{2} \mathrm{Cl}_{2}\right) ; v_{\max }$ (neat) $/ \mathrm{cm}^{-1}$ 2923, 2853, 1603, 1508, 1463, 1321, 1242, 1208, 1152, 1118, 
1029,$1012 ; \delta_{\mathrm{H}}\left(200 \mathrm{MHz}, \mathrm{CD}_{3} \mathrm{CN}\right) 7.39-7.22(10 \mathrm{H}, \mathrm{m}, 10 \times \mathrm{Bn} H)$ $7.06(1 \mathrm{H}, \mathrm{d}, J 10.0, \mathrm{ArCH}=\mathrm{CHS}), 6.75(1 \mathrm{H}, \mathrm{s}, \mathrm{Ar} H), 6.67(1 \mathrm{H}, \mathrm{d}$, $J$ 10.0, $\mathrm{ArCH}=\mathrm{CHS}), 6.62(1 \mathrm{H}, \mathrm{s}, \mathrm{Ar} H), 4.54(2 \mathrm{H}, \mathrm{d}, J 13.0$, $\left.\mathrm{NCH}_{2} \mathrm{Ph}\right), 4.30\left(2 \mathrm{H}, \mathrm{d}, J\right.$ 13.0, $\left.\mathrm{NCH}_{2} \mathrm{Ph}\right), 3.71(3 \mathrm{H}, \mathrm{s}, \mathrm{OMe}), 3.64$ $(3 \mathrm{H}, \mathrm{s}, \mathrm{OMe}) ; \delta_{\mathrm{C}}\left(126 \mathrm{MHz}, \mathrm{CDCl}_{3}\right) 152.5(\mathrm{C}), 146.5(\mathrm{C}), 138.5$ (C), $138.3(\mathrm{C}), 136.3(\mathrm{CH}), 130.8(4 \times \mathrm{CH}), 129.2(4 \times \mathrm{CH}), 128.7$ $(2 \times \mathrm{CH}), 126.4(\mathrm{C}), 122.9(\mathrm{CH}), 114.8(\mathrm{C}), 111.3(\mathrm{CH}), 104.0$ $(\mathrm{CH}), 60.7\left(2 \times \mathrm{CH}_{2}\right), 56.6\left(\mathrm{CH}_{3}\right), 56.5\left(\mathrm{CH}_{3}\right) ; \mathrm{m} / z\left(\mathrm{ESI}^{+}\right) 459([\mathrm{M}$ $\left.+\mathrm{Na}]^{+}, 100 \%\right)$; HMRS $\left(\mathrm{ESI}^{+}\right) \mathrm{C}_{24} \mathrm{H}_{24} \mathrm{~N}_{2} \mathrm{NaO}_{4} \mathrm{~S}^{+}\left([\mathrm{M}+\mathrm{Na}]^{+}\right)$ requires 459.1349 , found 459.1356 .

\subsubsection{6,7-Dimethoxy-1-(methyl(phenyl)amino)-1H- benzo[c][1,2] thiazine 2,2-dioxide (4n)}

Prepared according to the general procedure B using 2-(2bromo-4,5-dimethoxyphenyl)- $N$ '-methyl- $N{ }^{\prime}$ -

phenylethenesulfonohydrazide 3n (65 mg, $0.15 \mathrm{mmol}, Z: E, 10: 1)$. Column chromatography (eluent: $3: 7$, petrol:ether) yielded benzothiazine 2,2-dioxide $4 \mathbf{n}(22 \mathrm{mg}, 42 \%)$ as an off-white crystalline solid; $\mathrm{mp} 205-208{ }^{\circ} \mathrm{C}\left(\mathrm{CH}_{2} \mathrm{Cl}_{2}\right) ; v_{\max }$ (neat) $/ \mathrm{cm}^{-1} 2934$, $2867,1605,1510,1474,1245,1208,1152,1110 ; \delta_{\mathrm{H}}(200 \mathrm{MHz}$, $\left.\left(\mathrm{CD}_{3}\right)_{2} \mathrm{CO}\right) 7.46(1 \mathrm{H}, \mathrm{d}, J 10.0, \mathrm{ArCH}=\mathrm{CHS}), 7.19-6.55(8 \mathrm{H}, \mathrm{m}$, $\mathrm{ArCH}=\mathrm{CHS}, 7 \times \mathrm{ArH}), 3.83(3 \mathrm{H}, \mathrm{s}, \mathrm{OMe}), 3.63(3 \mathrm{H}, \mathrm{s}, \mathrm{OMe}), 2.83$ $(3 \mathrm{H}, \mathrm{s}, \mathrm{NMe}) ; \delta_{\mathrm{C}}\left(126 \mathrm{MHz},\left(\mathrm{CD}_{3}\right)_{2} \mathrm{CO}\right) 152.7(\mathrm{C}), 146.3(\mathrm{C})$, $135.7(\mathrm{CH}), 131.5(2 \times \mathrm{CH}), 129.6(\mathrm{C}), 126.0(2 \times \mathrm{CH}), 121.4$ $(\mathrm{CH}), 115.5(\mathrm{C}), 114.5(\mathrm{C}), 113.1(\mathrm{CH}), 112.6(\mathrm{CH}), 103.3(\mathrm{CH})$, $56.5\left(\mathrm{CH}_{3}\right), 56.1\left(\mathrm{CH}_{3}\right), 35.0\left(\mathrm{CH}_{3}\right) ; \mathrm{m} / z\left(\mathrm{ESI}^{+}\right) 369\left([\mathrm{M}+\mathrm{Na}]^{+}\right.$, $50 \%), 347\left([\mathrm{M}+\mathrm{H}]^{+}, 100 \%\right) ; \mathrm{HMRS}\left(\mathrm{ESI}^{+}\right) \mathrm{C}_{17} \mathrm{H}_{18} \mathrm{~N}_{2} \mathrm{NaO}_{4} \mathrm{~S}^{+}([\mathrm{M}$ $+\mathrm{Na}]^{+}$) requires 347.1060 , found 347.1066 .

4.4. (Z) and (E)-2-(2-Fluorophenyl)-N-(morpholin-4yl)ethenesulfonamide (Z)-6a, (E)-6a

Prepared according to the general procedure A using DABSO (63 mg, $0.28 \mathrm{mmol})$, CataCXium A (17 mg, $0.05 \mathrm{mmol}),(Z)-1-$ fluoro-2-(2-iodovinyl)benzene ( $69 \mathrm{mg}, 0.24 \mathrm{mmol}, Z: E,>20: 1)$, 4aminomorpholine $(35 \mu \mathrm{L}, 0.36 \mathrm{mmol})$ and 1,4-dioxane $(1.6 \mathrm{~mL})$. The reaction mixture was stirred at $70{ }^{\circ} \mathrm{C}$ for $8 \mathrm{~h}$. Column chromatography (eluent: $0-50 \%$, ether in hexane) yielded, in order of elution, alkenyl $N$-aminosulfonamide $(Z)-\mathbf{6 a}(31 \mathrm{mg}$, $45 \%$ ) and alkenyl $N$-aminosulfonamide $(E)-6 \mathbf{a}(8 \mathrm{mg}, 11 \%)$, as offwhite crystalline solids. (Z)-6a mp 96-98 ${ }^{\circ} \mathrm{C}\left(\mathrm{CH}_{2} \mathrm{Cl}_{2}\right) ; v_{\max }$ (neat) $/ \mathrm{cm}^{-1} 3212,2827,2777,1325,1311,1278,1265,1243,1211$, $1203,1170,1142,1100,1070,1029,1008 ; \delta_{\mathrm{H}}\left(400 \mathrm{MHz}, \mathrm{CDCl}_{3}\right)$ $7.95(1 \mathrm{H}$, app t, $J 8.0, \operatorname{Ar} H), 7.40(1 \mathrm{H}$, app q, $J 8.0, \operatorname{Ar} H), 7.26-$ $7.16(2 \mathrm{H}, \mathrm{m}, \mathrm{Ar} H, \mathrm{ArCH}=\mathrm{CHS}), 7.09(1 \mathrm{H}$, app t, $J 8.0, \operatorname{Ar} H), 6.58$ $(1 \mathrm{H}, \mathrm{d}, J 12.0, \mathrm{ArCH}=\mathrm{CHS}), 5.43(1 \mathrm{H}, \mathrm{s}, \mathrm{NH}), 3.72(4 \mathrm{H}, \mathrm{t}, J 4.5$, $\left.\mathrm{N}\left(\mathrm{CH}_{2} \mathrm{CH}_{2}\right)_{2} \mathrm{O}\right), 2.83$ (4H, br. t, $\left.J 4.5, \mathrm{~N}\left(\mathrm{CH}_{2} \mathrm{CH}_{2}\right)_{2} \mathrm{O}\right) ; \delta_{\mathrm{C}}(101$ $\left.\mathrm{MHz}, \mathrm{CDCl}_{3}\right) 160.5\left(\mathrm{C}, \mathrm{d}, J_{\mathrm{CF}} 249.5\right), 134.0\left(\mathrm{CH}, \mathrm{d}, J_{\mathrm{CF}} 5.0\right), 132.0$ $\left(\mathrm{CH}, \mathrm{d}, J_{\mathrm{CF}} 2.0\right), 131.9\left(\mathrm{CH}, \mathrm{d}, J_{\mathrm{CF}} 8.5\right), 128.8(\mathrm{CH}), 123.9(\mathrm{CH}, \mathrm{d}$, $\left.J_{\mathrm{CF}} 3.0\right), 120.8\left(\mathrm{C}, \mathrm{d}, J_{\mathrm{CF}} 13.0\right), 115.2\left(\mathrm{CH}, \mathrm{d}, J_{\mathrm{CF}} 21.0\right), 66.6(2 \times$ $\left.\mathrm{CH}_{2}\right), 57.1\left(2 \times \mathrm{CH}_{2}\right) ; \delta_{\mathrm{F}}\left(377 \mathrm{MHz}, \mathrm{CDCl}_{3}\right)-113.3(\mathrm{~s}, \mathrm{Ar} F)\left\{{ }^{1} \mathrm{H}\right\}$; $\mathrm{m} / z\left(\mathrm{ESI}^{+}\right) 595\left([2 \mathrm{M}+\mathrm{Na}]^{+}, 100 \%\right), 309\left([\mathrm{M}+\mathrm{Na}]^{+}, 42 \%\right) ; \mathrm{m} / \mathrm{z}$ (ESI $) 285\left([\mathrm{M}-\mathrm{H}]^{-}, 100 \%\right) ; \mathrm{HRMS}\left(\mathrm{ESI}^{+}\right) \mathrm{C}_{12} \mathrm{H}_{15} \mathrm{FN}_{2} \mathrm{NaO}_{3} \mathrm{~S}^{+}([\mathrm{M}$ $+\mathrm{Na}^{+}$) requires 309.0680, found 309.0675; Anal. Calcd. (\%) for $\mathrm{C}_{12} \mathrm{H}_{15} \mathrm{FN}_{2} \mathrm{O}_{3} \mathrm{~S}$ : C 50.34, H 5.28, N 9.78; found C 50.45, H 5.18, N 9.75. (E)-6a: $\mathrm{mp} 137-140{ }^{\circ} \mathrm{C}\left(\mathrm{CH}_{2} \mathrm{Cl}_{2}\right) ; v_{\max }$ (neat) $/ \mathrm{cm}^{-1} 3280$, 2917, 2850, 1368, 1333, 1318, 1293, 1264, 1238, 1221, 1138, 1106,$1032 ; \delta_{\mathrm{H}}\left(400 \mathrm{MHz}, \mathrm{CDCl}_{3}\right) 7.66(1 \mathrm{H}, \mathrm{d}, J 15.5$, $\mathrm{ArCH}=\mathrm{CHS}), 7.49(1 \mathrm{H}$, app t, $J$ 7.5, $\mathrm{ArH}), 7.46-7.38(1 \mathrm{H}, \mathrm{m}$, $\operatorname{Ar} H), 7.22(1 \mathrm{H}$, app t, $J 7.5, \operatorname{Ar} H), 7.19-7.11(1 \mathrm{H}, \mathrm{m}, \operatorname{Ar} H), 6.99$ $(1 \mathrm{H}, \mathrm{d}, J 15.5, \mathrm{ArCH}=\mathrm{CHS}), 5.45(1 \mathrm{H}, \mathrm{s}, \mathrm{NH}), 3.74(4 \mathrm{H}, \mathrm{t}, J 4.5$, $\left.\mathrm{N}\left(\mathrm{CH}_{2} \mathrm{CH}_{2}\right)_{2} \mathrm{O}\right), 2.89$ (4H, br. t, $\left.J 4.5, \mathrm{~N}\left(\mathrm{CH}_{2} \mathrm{CH}_{2}\right)_{2} \mathrm{O}\right) ; \delta_{\mathrm{C}}(101$ $\left.\mathrm{MHz}, \mathrm{CDCl}_{3}\right) 161.7\left(\mathrm{C}, \mathrm{d}, J_{\mathrm{CF}} 261.5\right), 137.0(\mathrm{CH}), 132.7(\mathrm{CH}, \mathrm{d}$, $\left.J_{\mathrm{CF}} 9.0\right), 130.5\left(\mathrm{CH}, \mathrm{d}, J_{\mathrm{CF}} 3.0\right), 126.8\left(\mathrm{CH}, \mathrm{d}, J_{\mathrm{CF}} 9.5\right), 124.9(\mathrm{CH}$, $\left.\mathrm{d}, J_{\mathrm{CF}} 5.0\right), 120.9\left(\mathrm{C}, \mathrm{d}, J_{\mathrm{CF}} 11.0\right), 116.6\left(\mathrm{CH}, \mathrm{d}, J_{\mathrm{CF}} 21.5\right), 66.7$ $\left(2 \times \mathrm{CH}_{2}\right), 57.5\left(2 \times \mathrm{CH}_{2}\right) ; m / z\left(\mathrm{ESI}^{+}\right) 595\left([2 \mathrm{M}+\mathrm{Na}]^{+}, 100 \%\right)$, $309\left(\left[\mathrm{M}+\mathrm{Na}^{+}, 42 \%\right) ; \mathrm{m} / z\left(\mathrm{ESI}^{-}\right) 285.1\left([\mathrm{M}-\mathrm{H}]^{-}, 100 \%\right) ; \delta_{\mathrm{F}}\right.$ $\left(377 \mathrm{MHz}, \mathrm{CDCl}_{3}\right)-112.6(\mathrm{~s}, \mathrm{Ar} F) \quad\left\{{ }^{1} \mathrm{H}\right\} ; \mathrm{HRMS}\left(\mathrm{ESI}^{+}\right)$ $\mathrm{C}_{12} \mathrm{H}_{15} \mathrm{FN}_{2} \mathrm{NaO}_{3} \mathrm{~S}^{+}\left([\mathrm{M}+\mathrm{Na}]^{+}\right)$requires 309.0680 , found 309.0675 .

4.5 (Z)-2-[2-Fluoro-5-(trifluoromethyl)phenyl]-N-(morpholin4-yl)ethenesulfonamide $\mathbf{6} \boldsymbol{b}$

Prepared according to the general procedure A using DABSO (126 mg, $0.53 \mathrm{mmol}$ ), CataCXium A (34 mg, $0.10 \mathrm{mmol}),(Z)-1$ fluoro-2-(2-iodovinyl)-4-(trifluoromethyl)benzene (152 mg, 0.24 $\mathrm{mmol}, Z: E,>20: 1)$ and 4-aminomorpholine $(69 \mu \mathrm{L}, 0.72 \mathrm{mmol})$. The reaction mixture was stirred at $70{ }^{\circ} \mathrm{C}$ for $6 \mathrm{~h}$. Column chromatography (eluent: 7:3, ether:petrol) yielded alkenyl $N$-aminosulfonamide $\mathbf{6 b}$ as a pale yellow crystalline solid (38 $\mathrm{mg}$, $45 \%, Z: E,>20: 1) ; \mathrm{mp} 87-89^{\circ} \mathrm{C}\left(\mathrm{CH}_{2} \mathrm{Cl}_{2}\right) ; v_{\max }$ (neat) $/ \mathrm{cm}^{-1} 3200$, 2963, 1635, 1499, 1459, 1329, 1287, 1259, 1208, 1105, 1073, $1015 ; \delta_{\mathrm{H}}\left(500 \mathrm{MHz}, \mathrm{CDCl}_{3}\right) 8.28(1 \mathrm{H}, \mathrm{dd}, J$ 7.0, $2.0 \mathrm{ArH})$, 7.67-7.62 (1H, m, ArH), 7.24-7.16 (2H, m, ArH, ArCH=CHS), $6.58(1 \mathrm{H}, \mathrm{d}, J 12.0, \mathrm{ArCH}=\mathrm{CHS}), 5.70(1 \mathrm{H}, \mathrm{s}, \mathrm{NH}), 3.73(4 \mathrm{H}, \mathrm{t}, J$ $\left.4.5, \mathrm{~N}\left(\mathrm{CH}_{2} \mathrm{CH}_{2}\right)_{2} \mathrm{O}\right), 2.88\left(4 \mathrm{H}\right.$, br. t, $\left.J 4.5, \mathrm{~N}\left(\mathrm{CH}_{2} \mathrm{CH}_{2}\right)_{2} \mathrm{O}\right) ; \delta_{\mathrm{C}}(126$ $\left.\mathrm{MHz}, \mathrm{CDCl}_{3}\right) 162.0\left(\mathrm{C}, \mathrm{d}, J_{\mathrm{CF}} 255.5\right), 132.8\left(\mathrm{CH}, \mathrm{d}, J_{\mathrm{CF}} 4.0\right), 130.1$ $(\mathrm{CH}), 129.9\left(\mathrm{CH}\right.$, app quin, $\left.J_{\mathrm{CF}} 4.0\right), 128.8\left(\mathrm{CH}, \mathrm{dq}, J_{\mathrm{CF}} 9.5,4.0\right)$, $126.4\left(\mathrm{CH}, \mathrm{qd}, J_{\mathrm{CF}} 30.0,4.0\right), 123.7\left(\mathrm{C}, \mathrm{q}, J_{\mathrm{CF}} 272.5\right), 121.3(\mathrm{C}, \mathrm{d}$, $\left.J_{\mathrm{CF}} 14.5\right), 115.9\left(\mathrm{CH}, \mathrm{d}, J_{\mathrm{CF}} 23.0\right), 66.6\left(2 \times \mathrm{CH}_{2}\right), 57.4\left(2 \times \mathrm{CH}_{2}\right)$; $\delta_{\mathrm{F}}\left(377 \mathrm{MHz}, \mathrm{CDCl}_{3}\right)-61.8\left(\mathrm{~s}, \mathrm{ArCF}_{3}\right),-107.9(\mathrm{~s}, \mathrm{Ar} F)\left\{{ }^{1} \mathrm{H}\right\} ; \mathrm{m} / z$ $\left(\mathrm{ESI}^{+}\right) 731([2 \mathrm{M}+\mathrm{Na}], 98 \%), 377\left([\mathrm{M}+\mathrm{Na}]^{+}, 100 \%\right), 355([\mathrm{M}+$ $\left.\mathrm{H}]^{+}, 12 \%\right) ; \mathrm{m} / z\left(\mathrm{ESI}^{-}\right) 353\left([\mathrm{M}-\mathrm{H}]^{-}, 100 \%\right) ; \mathrm{HRMS}\left(\mathrm{ESI}^{+}\right)$ $\mathrm{C}_{13} \mathrm{H}_{14} \mathrm{~F}_{4} \mathrm{~N}_{2} \mathrm{NaO}_{3} \mathrm{~S}^{+}\left([\mathrm{M}+\mathrm{Na}]^{+}\right)$requires 377.0553, found 377.0544 .

4.6 1-(Morpholin-4-yl)-6-(trifluoromethyl)-1H-2,1benzothiazine 2,2-dioxide (4b) using $S_{N} A r$ chemistry

2-[2-Fluoro-5-(trifluoromethyl)phenyl]- $N$-(morpholin-4yl)ethenesulfonamide $\mathbf{6 b}(30 \mathrm{mg}, 0.08 \mathrm{mmol}, Z: E,>20: 1)$ was added to a mixture of $\mathrm{Cs}_{2} \mathrm{CO}_{3}(83 \mathrm{mg}, 0.24 \mathrm{mmol})$ and 1,4-dioxane $(0.5 \mathrm{~mL})$. The reaction mixture was stirred at $100^{\circ} \mathrm{C}$ for $1 \mathrm{~h}$. The reaction mixture was cooled to $\mathrm{RT}$ and filtered through a short pad of Celite with $\mathrm{CH}_{2} \mathrm{Cl}_{2}(5 \mathrm{~mL})$ and ether $(5 \mathrm{~mL})$. Column chromatography (eluent: 3:7, petrol:ether) yielded benzothiazine 2,2-dioxide $\mathbf{4 b}$ as a white crystalline solid (24 mg, 88\%). Data as above.

4.7 1-Morpholino-3,4-dihydro-1H-benzo[c][1,2]thiazine 2,2dioxide 7

To a solution of 1-(morpholin-4-yl)-1H-2,1-benzothiazine 2,2dioxide $4 \mathbf{a}(53 \mathrm{mg}, 0.20 \mathrm{mmol})$ in degassed ethanol $(5 \mathrm{~mL})$ was added $\mathrm{Pd} / \mathrm{C} 10$ wt.\% (5 mg) and the flask flushed with $\mathrm{H}_{2}$. The reaction mixture was stirred at $\mathrm{RT}$ under a $\mathrm{H}_{2}$ balloon for $3 \mathrm{~h}$. The flask was flushed with $\mathrm{N}_{2}$ and the suspension filtered through Celite and washed with ethanol. The filtrate was concentrated in vacuo to yield sulfonamide 7 as a white crystalline solid (49 mg, 91\%); mp $270{ }^{\circ} \mathrm{C}(\mathrm{dec})\left(\mathrm{CH}_{2} \mathrm{Cl}_{2}\right) ; v_{\max }$ (neat) $/ \mathrm{cm}^{-1} 2924,2857$, 1434, 1452, 1337, 1294, 1269, 1160, 1134, 1110; $\delta_{\mathrm{H}}(500 \mathrm{MHz}$, $\left.\mathrm{CDCl}_{3}\right) 7.52(1 \mathrm{H}, \mathrm{dd}, J 8.5,1.0, \mathrm{Ar} H), 7.29-7.24(1 \mathrm{H}, \mathrm{m}, \mathrm{Ar} H)$, $7.13(1 \mathrm{H}, \mathrm{d}, J 7.5, \mathrm{ArH}), 7.04$ (1H, app td, J 7.5, 1.0, $\mathrm{ArH}), 3.79$ $\left(4 \mathrm{H}, \mathrm{t}, \mathrm{J} 4.5, \mathrm{~N}\left(\mathrm{CH}_{2} \mathrm{CH}_{2}\right)_{2} \mathrm{O}\right), 3.47-3.41\left(6 \mathrm{H}, \mathrm{m}, \mathrm{N}\left(\mathrm{CH}_{2} \mathrm{CH}_{2}\right)_{2} \mathrm{O}\right.$, $\left.\mathrm{ArCH}_{2} \mathrm{CH}_{2} \mathrm{~S}\right), 3.37-3.33\left(2 \mathrm{H}, \mathrm{m}, \mathrm{ArCH}_{2} \mathrm{CH}_{2} \mathrm{~S}\right) ; \delta_{\mathrm{C}}(126 \mathrm{MHz}$, $\left.\mathrm{CDCl}_{3}\right) 141.3(\mathrm{C}), 129.5(\mathrm{CH}), 128.0(\mathrm{CH}), 123.7(\mathrm{CH}), 122.1(\mathrm{C})$, $118.4(\mathrm{CH}), 68.1\left(2 \times \mathrm{CH}_{2}\right), 53.2\left(2 \times \mathrm{CH}_{2}\right), 47.9\left(\mathrm{CH}_{2}\right), 28.3$ $\left(\mathrm{CH}_{2}\right) ; \mathrm{m} / z\left(\mathrm{ESI}^{+}\right) 291\left([\mathrm{M}+\mathrm{Na}]^{+}, 100 \%\right), 269\left([\mathrm{M}+\mathrm{H}]^{+}, 20 \%\right)$; 
HMRS $\left(\mathrm{ESI}^{+}\right) \mathrm{C}_{12} \mathrm{H}_{17} \mathrm{~N}_{2} \mathrm{O}_{3} \mathrm{~S}^{+}\left([\mathrm{M}+\mathrm{H}]^{+}\right)$requires 269.0954, found 269.0959 .

4.8 6,7-Dimethoxy-3,4-dihydro-1H-benzo[c][1,2]thiazine 2,2dioxide 8

To a solution of 1-(dibenzylamino)-6,7-dimethoxy-1Hbenzo[c][1,2]thiazine 2,2-dioxide $4 \mathrm{~m}(45 \mathrm{mg}, 0.10 \mathrm{mmol})$ in degassed acetone $(0.3 \mathrm{~mL})$ was added Pearlman's catalyst $(9 \mathrm{mg}$, 20 wt. $\% 60 \%$ moisture, $\left.\operatorname{Pd}\left(\mathrm{OH}_{2}\right) / \mathrm{C}\right)$ and the reaction flask was flushed with $\mathrm{H}_{2}$. The reaction mixture was stirred at $\mathrm{RT}$ under a $\mathrm{H}_{2}$ balloon for $16 \mathrm{~h}$. The flask was then flushed with $\mathrm{N}_{2}$ and the suspension filtered through Celite and washed with acetone. The filtrate was concentrated in vacuo. Acetic acid $(1.1 \mathrm{~mL})$ and zinc dust $(17 \mathrm{mg}, 2.60 \mathrm{mmol})$ was added and the reaction mixture stirred for $2 \mathrm{~h}$ at RT. The reaction mixture was diluted with $\mathrm{CH}_{2} \mathrm{Cl}_{2}$ $(5 \mathrm{~mL})$ and the suspension filtered through Celite. Water was added and the organic layer extracted with $\mathrm{CH}_{2} \mathrm{Cl}_{2}$. The combined organic layers were washed with brine, dried over $\mathrm{MgSO}_{4}$, filtered and concentrated in vacuo. Column chromatography (eluent: ether) yielded sulfonamide $\mathbf{8}$ as a white crystalline solid (16 mg, $68 \%) ; \mathrm{mp} 145-148^{\circ} \mathrm{C}\left(\mathrm{CH}_{2} \mathrm{Cl}_{2}\right) ; v_{\max }$ (neat) $/ \mathrm{cm}^{-1} 3225,2925,2850$, $1605,1503,1463,1325,1270,1152,1121,1111 ; \delta_{\mathrm{H}}(500 \mathrm{MHz}$, $\left.\mathrm{CDCl}_{3}\right) 6.64(1 \mathrm{H}, \mathrm{s}, \mathrm{Ar} H), 6.31(1 \mathrm{H}, \mathrm{s}, \mathrm{Ar} H), 6.01(1 \mathrm{H}, \mathrm{s}, \mathrm{N} H)$, $3.85(3 \mathrm{H}, \mathrm{s}, \mathrm{OMe}), 3.84(3 \mathrm{H}, \mathrm{s}, \mathrm{OMe}), 3.43-3.37\left(2 \mathrm{H}, \mathrm{m}, \mathrm{CH}_{2}\right)$, 3.31-3.26 $\left(2 \mathrm{H}, \mathrm{m}, \mathrm{CH}_{2}\right) ; \delta_{\mathrm{C}}\left(126 \mathrm{MHz}, \mathrm{CDCl}_{3}\right)$ 149.9, 146.2, $129.9,113.4,112.2,104.0,56.4,56.2,45.9,28.2$; HMRS (ESI') $\mathrm{C}_{10} \mathrm{H}_{12} \mathrm{NO}_{4} \mathrm{~S}^{-}\left([\mathrm{M}-\mathrm{H}]^{-}\right)$requires 242.0493 , found 242.0495 .

\section{Acknowledgements}

The authors gratefully acknowledge the EPSRC and Pfizer for support of this work.

\section{Appendix A. Supplementary data}

Supplementary data relating to this article (NMR spectra) may be found at XXXXXXX.

\section{References}

a) S. Mondal, S. Malakar, Tetrahedron 2020, 76; b) K. A. Scott, J. T. Njardarson, Top. Curr. Chem. (Z) 2018, 376; c) A. S. Kalgutkar, R. Jones, A. Sawant, in Metabolism, Pharmacokinetics and Toxicity of Functional Groups: Impact of the Building Blocks of Medicinal Chemistry on Admet (Ed.: D. A. Smith), Royal Soc Chemistry, Cambridge, 2010, pp. 210-274. C. Yoakim, J. O’Meara, B. Simoneau, W.. W. Ogilvie, R. Deziel, WO 2004026875, 2004. .

3 H. Nie, K. L. Widdowson, WO 9834929, 1998.

4 W. Li, C. K. Marlowe, R. M. Scarborough, WO 2001072725, 2001

5 a) B. Loev, M. F. Kormendy, K. M. Snader, J. Org. Chem. 1966, 31, 3531-3534; b) S. Rossi, G. Pagani, Ann. Chim.Rome 1966, 56, 741.

6 a) J. Minville, J. Poulin, C. Dufresne, C. F. Sturino, Tetrahedron Lett. 2008, 49, 3677-3681; b) P. R. Hanson, D. A. Probst, R. E. Robinson, M. Yau, Tetrahedron Lett. 1999, 40, 4761-4764.

7 a) C. B. Bheeter, J. K. Bera, H. Doucet, Adv. Synth. Catal. 2012, 354, 3533-3538; b) Y. Li, Q. Ding, G. Qiu, J. Wu, Org. Biomol. Chem. 2014, 12, 149-155; c) R. A. Abramovitch, K. Miyashita, Journal of the Chemical Society, Perkin Transactions 1 1975, 2413-2416. a) H. Togo, T. Nabana, K. Yamaguchi, J. Org. Chem. 2000, 65, 8391-8394; b) A. Fensome, J. Goldberg, C. C. McComas, E. J. Trybulski, R. P. Woodworth, D. C.
Deecher, G. T. Whiteside, P. Zhang, Bioorg Med Chem Lett 2010, 20, 1555-1558.

a) G. M. Coppola, J. Het. Chem. 1981, 18, 845-846; b) G. M. Coppola, G. E. Hardtmann, J. Het. Chem. 1979, 16, 1361-1363.

a) A. C. Tadd, A. Matsuno, M. R. Fielding, M. C. Willis, Org. Lett. 2009, 11, 583-586; b) A. C. Tadd, M. R. Fielding, M. C. Willis, Chem. Commun. 2009, 6744.

a) B. Nguyen, E. J. Emmett, M. C. Willis, J. Am. Chem. Soc. 2010, 132, 16372-16373; b) E. J. Emmett, C. S. RichardsTaylor, B. Nguyen, A. Garcia-Rubia, B. R. Hayter, M. C. Willis, Org. Biomol. Chem. 2012, 10, 4007-4014; c) E. J. Emmett, B. R. Hayter, M. C. Willis, Angew. Chem. Int. Ed. 2014, 53, 10204-10208; d) C. S. Richards-Taylor, D. C. Blakemore, M. C. Willis, Chem. Sci. 2014, 5, 222-228; e) A. S. Deeming, C. J. Russell, M. C. Willis, Angew. Chem. Int. Ed. 2016, 55, 747-750; f) A. T. Davies, J. M. Curto, S. W. Bagley, M. C. Willis, Chem Sci 2017, 8, 1233-1237; g) T. S. Lou, S. W. Bagley, M. C. Willis, Angew. Chem. Int. Ed. 2019, 58, 18859-18863.

12 a) S. Ye, J. Wu, Chem. Commun. 2012, 48, 7753-7755; b) S. Q. Ye, J. Wu, Chem. Commun. 2012, 48, 10037-10039; c) A. Shavnya, S. B. Coffey, A. C. Smith, V. Mascitti, Org. Lett. 2013, 15, 6226-6229; d) A. Shavnya, K. D. Hesp, V. Mascitti, A. C. Smith, Angew. Chem. Int. Ed. 2015, 54, 13571-13575; e) Y. Y. An, H. G. Xia, J. Wu, Org. Biomol. Chem. 2016, 14, 1665-1669; f) H. Konishi, H. Tanaka, K. Manabe, Org. Lett. 2017, 7, 1578-1581.

H. Woolven, C. González-Rodríguez, I. Marco, A. L. Thompson, M. C. Willis, Org. Lett. 2011, 13, 4876-4878.

a) M. C. Willis, G. N. Brace, T. J. K. Findlay, I. P. Holmes, Adv. Synth. Catal. 2006, 348, 851-856; b) C. J. Ball, J. Gilmore, M. C. Willis, Angew. Chem. Int. Ed. 2012, 51, 5718-5722. 